\title{
Movement curvature planning through force field internal models
}

\author{
Biljana Petreska · Aude Billard
}

Received: 18 September 2008 / Accepted: 16 February 2009 / Published online: 21 April 2009

(C) Springer-Verlag 2009

\begin{abstract}
Human motion studies have focused primarily on modeling straight point-to-point reaching movements. However, many goal-directed reaching movements, such as movements directed towards oneself, are not straight but rather follow highly curved trajectories. These movements are particularly interesting to study since they are essential in our everyday life, appear early in development and are routinely used to assess movement deficits following brain lesions. We argue that curved and straight-line reaching movements are generated by a unique neural controller and that the observed curvature of the movement is the result of an active control strategy that follows the geometry of one's body, for instance to avoid trajectories that would hit the body or yield postures close to the joint limits. We present a mathematical model that accounts for such an active control strategy and show that the model reproduces with high accuracy the kinematic features of human data during unconstrained reaching movements directed toward the head. The model consists of a nonlinear dynamical system with a single stable attractor at the target. Embodiment-related task constraints are expressed as a force field that acts on the dynamical system. Finally, we discuss the biological plausibility and neural correlates of the model's parameters and suggest that embodiment should be considered as a main cause for movement trajectory curvature.
\end{abstract}

Keywords Motor control - Neural control of movement . Dynamical systems · Computational model · Goal-directed reaching movements

\footnotetext{
B. Petreska $\cdot$ A. Billard $(\bowtie)$

Learning Algorithms and Systems Laboratory,

Ecole Polytechnique Fédérale de Lausanne,

EPFL-STI-I2S-LASA, Station 9,

1015 Lausanne, Switzerland

e-mail: biljana.petreska@a3.epfl.ch
}

\section{Introduction}

The vast majority of motor control studies have focused on highly constrained reaching movements, limiting the movements to a two-dimensional plane, and in particular to the frontal plane. These constraints are meant to ensure the reproducibility and controllability of the task. They have led to the observation of so-called "quasi-straight" reaching movements with a stereotyped single-peaked, bell-shaped velocity profile (Morasso 1981; Flash and Hogan 1985). The gentle curvature responsible for the term "quasi" has proved hard to explain. Some have suggested that it is due to distortions in the visual perception of the target (Wolpert et al. 1994, 1995), which could however not explain the fact that these are also observed in congenitally blind subjects (de Graaf et al. 1994). Others have attributed the curvature of the movement to the dynamics of the arm's biomechanics, i.e., inertial and viscoelastic resistive forces (Flash 1987; Bullock and Grossberg 1988). This again could not explain the fact that the curvature persists in isometric tasks, which indicates rather that the curvature is encoded directly in the activation patterns of the muscles (Pellegrini and Flanders 1996). Another possible explanation for the curvature of arm movements is Listing's law, as the arm rotation movements were shown to roughly lie in a $2 \mathrm{D}$ curved surface (Liebermann et al. 2006). Importantly, when participants are instructed to generate straight paths, they produce movements much straighter than those generated spontaneously (de Graaf et al. 1994; Desmurget et al. 1997; Osu et al. 1997), which argues against the hypothesis of imperfect control (Flash and Hogan 1985). In addition, the curvature depends on the location of the target (Soechting and Lacquaniti 1981) and is systematic within trials and across subjects (Soechting and Lacquaniti 1981; Pellegrini and Flanders 1996). Curved trajectories are also more frequently observed during unconstrained movements 


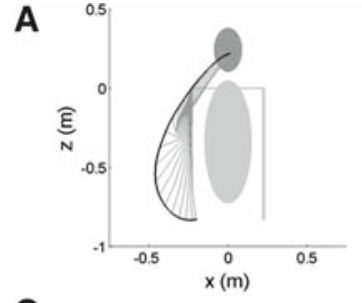

C
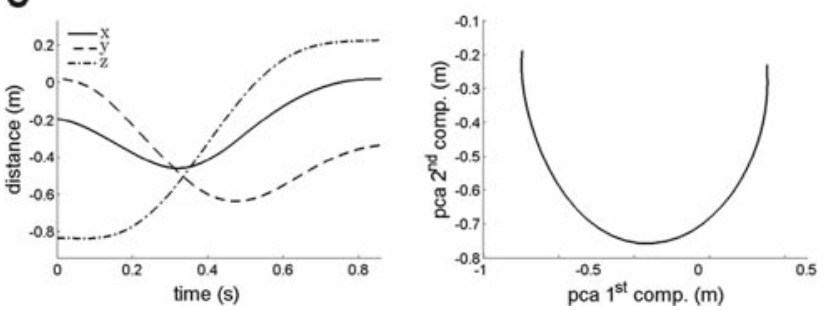

Fig. 1 An example of the curvature of an unconstrained self-oriented movement (the subject was asked to touch his nose). a Projections of the movement in the $x y-, x z-$, and $y z$-planes. $\mathbf{b}$ The velocity profile is bellshaped and single-peaked, similarly to the velocity profiles of straight point-to-point movements. $\mathbf{c}$ The movement is curved in the extrinsic hand Cartesian space (left), which is best visible when projected on the

(Soechting and Lacquaniti 1981; Lacquaniti et al. 1986; Miall and Haggard 1995; Desmurget et al. 1997; Osu et al. 1997). Overall, the above evidence indicates that the curvature underlying human motion might be a "natural" feature of the movement, and the observed straightness an artifact of the restricted workspace.

We show in this paper that these non-linearities are particularly important when considering reaching movements directed to ourselves (see Fig. 1). Self-oriented movements are part of our daily repertoire (e.g., to eat). They are among the first to emerge in life and are likely the result of evolutionary old neural structures. Their study may thus reveal basic neural processes of motor control. For instance, electrical stimulation of the precentral and motor cortices evoked natural multijoint movements that reached to different points in space, such as for example characteristic hand-to-mouth movements (Graziano et al. 2002, 2005). These movements are also routinely used in neurological examinations to test and diagnose various movement deficits following brain lesion (De Renzi and Lucchelli 1988; Goldenberg and Hagmann 1997; Petreska et al. 2007), which directly inspired the stimuli used in our study. All in all, the study of reaching movements toward oneself is particularly interesting from both a behavioral and a neurological perspective.

We will argue that movement curvature is planned by the central nervous system (CNS) and takes into account the geometry of the body. The idea that embodiment can be encapsulated in the control system itself is in line with our earlier observation that differences in the kinematic features of reaching movements in macaques and humans could be related to the biomechanical properties of the macaques' and
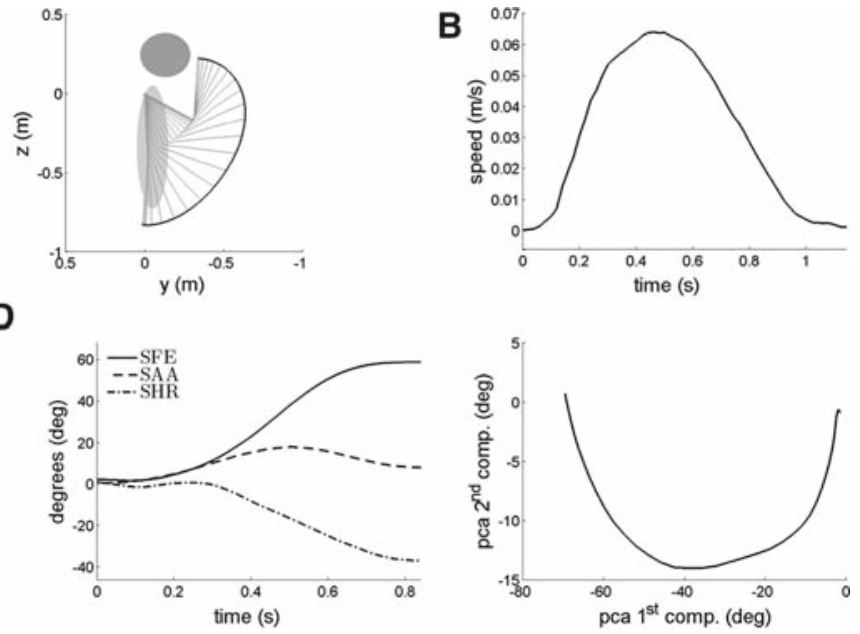

first two principal components following a principal component analysis (PCA) (right). d The movement is curved also in the intrinsic joint angles space (left) and its two principal components (right). The joint angles represented here correspond to the three degrees of freedom of the shoulder: shoulder flexion-extension $(S F E)$, shoulder abductionadduction $(S A A)$ and shoulder humeral rotation $(S H R)$

humans' shoulder joints (Christel and Billard 2002). Importantly, the model proposed here is not limited to self-oriented movements and can be applied to any point-to-point reaching movement such as for example reaching to targets in the extrapersonal frontal workspace.

\section{Computational approach}

Modeling studies are particularly useful for distinguishing among all of the plausible mechanisms to encode movements, as long as their predictions are tested and validated against empirical behavioral or neurophysiological data.

However, existing models are unsuccessful at reproducing the curvature of natural human movements (Admiraal et al. 2004), up to several exceptions (Torres and Zipser 2002; Biess et al. 2007; Guigon et al. 2007). For instance, while the so-called 2/3 power law (Lacquaniti et al. 1983) could account well for the curvature observed during handwriting and drawing motions, it was unsuccessful at explaining the curvature of reaching movements in the 3-dimensional space (Schaal and Sternad 2001), including the movements we consider in this paper as shown on Fig. 2. Furthermore, the minimum work model (Soechting et al. 1995) successfully reproduces the final joint postures of pointing movements starting from different initial joint postures, but does not explain the time dependency across joint trajectories. A kinematic model that intrinsically constrains the arm joints according to Listing's law (i.e., such that the arm rotation vectors lie in a 2-dimensional surface) was partially successful at describing the experimental data (Liebermann et al. 


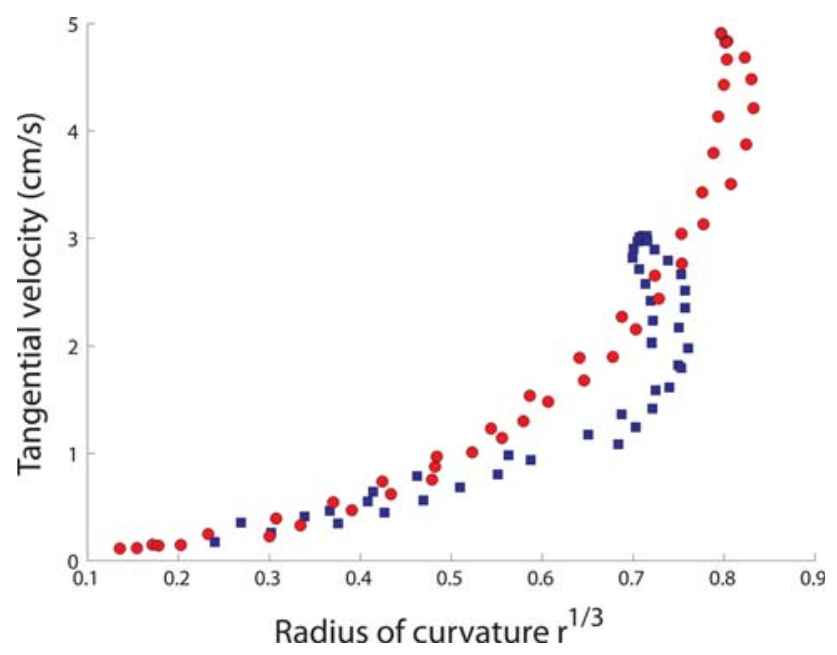

Fig. 2 Two examples of unconstrained self-oriented movements where the $2 / 3$ power law was degraded. The tangential velocity versus radius of curvature to the power $1 / 3$ is shown. The subject was asked to touch his nose (circles) or to touch his left ear (squares)

2006). The minimum hand jerk ${ }^{1}$ model (Flash and Hogan 1985) maximizes the smoothness of the hand trajectory in the extrinsic space. The result is a straight-line trajectory, whereas curved trajectories are obtained by specifying viapoints (e.g. for avoiding obstacles). However, it predicts a bimodal velocity profile which is at odds with the experimental data (Atkeson and Hollerbach 1985). Later it was suggested that the hand trajectory is the result of a compromise between planning a straight line in the task space and planning a straight line in the joint space (Cruse and Brüwer 1987; Okadome and Honda 1999; Hersch and Billard 2007). Such hybrid computations offer numerous advantages for controlling 3-dimensional reaching movements, such as avoiding singularities and avoiding hitting the joint limits (Hersch and Billard 2007). Unfortunately there is currently no direct neurophysiological evidence in support of such a control strategy. It has also been proposed that arm movements are controlled by minimizing the derivative of joint torques (Uno et al. 1989; Nakano et al. 1999; Wada et al. 2001). However, this model overestimates the magnitude of curvature of pointing movements (Biess et al. 2007). In Torres and Zipser (2002), the hand path is computed in the intrinsic joint angles space by minimizing an energy-like quantity, giving realistic predictions for curved paths. However, this model assumes a separate processing for the spatial and temporal dynamics of motion and displays some imprecisions for movements similar to those addressed here. The model by Biess et al. (2007) computes a geometrical joint angles geodesic path with respect to a kinetic energy metric in the Riemannian configuration space and subsequently

\footnotetext{
1 The jerk corresponds to the derivative of the acceleration and is a measure of the smoothness of the trajectory.
}

minimizes the squared jerk along this path. This model also treats the spatial and temporal dimensions separately and predicts identical path trajectories for different speeds. We find it difficult to evaluate how well this model would predict highly curved reaching movements as the pointing movements addressed in the study were quasi-straight, but we could observe that the model has difficulties with reproducing mixed curvatures (i.e., movements that deviate first to one side and then to the other side of the idealized straight trajectory). Another class of reaching models are stochastic models that take into account the noise inherent to the motor system. It has been consistently observed that the standard deviation of neuromotor commands increases with its mean (Sutton and Sykes 1967; Schmidt et al. 1979; Clamman 1969; Matthews 1996; St-Amant et al. 1998; Clancy and Hogan 1999; Osu et al. 2004). In line with this evidence, it was suggested that the brain minimizes the variance of the final arm position in the presence of such signal-dependent motor noise (Harris and Wolpert 1998; Hamilton et al. 2002). Even though this model succeeds at reproducing the curvature of 2-dimensional reaching movements, it does not specify which control laws generate these movements. In Todorov and Jordan (2002), an optimal feedback theory of motor control is proposed, in which the variability of the movement is distributed optimally among different degrees of freedom that do not interfere with the task goal. This qualitative model is appropriate for explaining the variability observed in reaching movements, it is however imprecise in its prediction of the curvature of movements. This is partly due to the determination of the appropriate cost function to optimize. This performance criterion is chosen arbitrarily and varies with the task. Another model based on the optimal feedback control theory was successful at reproducing the joint and hand trajectories of 3-dimensional movements (Guigon et al. 2007), but the authors admit that the movements reproduced are rather stereotyped. For example the model does not account for nonsymmetric velocity profiles or avoidance of extreme joint limits.

While it has been suggested that two different control strategies underlie straight and curved reaching movements (Desmurget et al. 1997; Moran and Schwartz 1999), we argue that these two types of movements are generated by a unique adaptive control mechanism. While none of the existing models offers a satisfactory solution for modeling the highly variable curvature of human movements, here we propose a dynamical model that accounts for both gently and highly curved hand trajectories, consistent with recent neurophysiological findings. First, unlike many of the models above, our model is closed-loop. Closed-loop control takes into account the uncertainty of the "real-world" and allows intelligent online corrections as well as robust responses to perturbations, rather than "playing a prerecorded tape" (Todorov 2004). Such an approach is in agreement with the observa- 
tion that the CNS is able to estimate and anticipate the state of the limb. This is achieved by integrating delayed sensory input and motor output through afferent and efferent internal feedback loops (Desmurget and Grafton 2000). The state information is used to continuously update the motor commands, which is likely to occur in the posterior parietal cortex and cerebellum.

Our model also takes advantage of the signal-dependant neuromotor noise mentioned earlier, which may be responsible for the speed-accuracy trade-off known as Fitts' law (Fitts 1954) and trail-to-trial variability (Todorov 2004). Finally, our model hypothesizes that the curvature of the hand trajectories is not an undesirable noise on otherwise perfect straight-line reaching movements. Rather, it is necessary and planned as such by the CNS in order to, for example, avoid impossible trajectories that go through the body and uncomfortable joint limit postures.

\section{Model description}

Our work was driven by the assumption that (a) a unique controller underlies both straight and curved reaching movements, and (b) that this controller is such that all the variables can be accounted for by known neurophysiological processes. Thus, to start with, we considered the vector integration to endpoint (VITE) model for point-to-point reaching (Bullock and Grossberg 1988) that accounts for typical kinematic features of human reaching movements such as bellshaped velocity profiles and speed-accuracy trade-off. The model has been used to explain control in both hand extrinsic and joints intrinsic spaces (Ajemian et al. 2001; Hersch and Billard 2007). Most importantly, the dynamics of the VITE model's response displays a profile of activity similar to that of populations of neurons in the primate's brain. In particular, the model could account for these neurons' sensitivity to change in the velocity of the movement and for the latency of activity at the movement onset (Bullock et al. 1998). The VITE model, however, suffers from a major restriction: it can generate only straight movements. ${ }^{2}$ Next, we describe the VITE model and give a formal definition of our extension that accounts for curved reaching movements.

\footnotetext{
2 An extension of the VITE model has been proposed to account for highly curved handwriting movements (Bullock et al. 1993; Paine et al. 2004), where three coupled VITE models control the displacement of the hand in a 2-dimensional plane and the rotation of the wrist. The curvature results from the coupling between the three models and the fact that each model is initiated with a slight delay at onset. This approach is not optimal for modeling simple point-to-point reaching movements as it necessitates the characterization of a sequence of multiple arbitrary targets, one for each change in the curvature.
}

\subsection{The original VITE model}

The original VITE model is a dynamic controller that at each point in time reduces the distance between the estimated and desired states of the controlled variable. First, it computes the desired movement acceleration based on the difference between the present and endpoint vectors. Second, this acceleration is integrated and primed with a faster-thanlinear time-dependent "go signal" to specify the desired speed, which is the control signal sent to the muscle motoneurons. This priming signal is essential for the obtention of a bell-shaped velocity profile.

In its complete form the VITE model succeeds for example at: maintaining accurate proprioception while controlling voluntary reaches to spatial targets, maintaining postures despite perturbations, complying with an imposed movement, exerting force against obstacles, compensating for static and inertial loads and reproducing muscle vibration effects (Cisek et al. 1998). For simplicity, we only use the concise form of the model presented in Bullock and Grossberg (1988). For a description of the original VITE model please see the Appendix.

\subsection{Modification of the original VITE model}

Our modified VITE system is governed by a non-linear and noisy spring-damper system given by:

$$
\ddot{\mathbf{x}}(t)=\overbrace{-\alpha \dot{\mathbf{x}}(t)}^{\text {damping factor }}+\overbrace{\beta \mathbf{g}\left(\mathbf{x}^{*}(t)-\mathbf{x}(t)+\eta\right)}^{\text {noisy endpoint attractor }} \mathbf{u}(t)
$$

The first term is a damping factor proportional to the speed $\dot{\mathbf{x}}(t)$ of the end-effector that prevents the system from oscillating too importantly. The second term corresponds to an elastic force that drives the end-effector from its actual position $\mathbf{x}(t)$ toward the desired target position $\mathbf{x}^{*}(t)$. Note that the desired position is written as a function of time in order to emphasize the ability of the system to track the target in real time without any additional computation (as a result the system is robust to perturbations of the target position). $\alpha$ is a time constant set to 50. $\beta \in \mathbb{R}^{+}$determines the amplitude of the speed at which the system moves globally (increasing $\beta$ would result in a higher velocity peak and shorter movement duration, see Fig. 3a). g is a nonlinear function that modulates the dynamics of the system so that it presents a typical bell-shaped velocity profile (refer to the Appendix for the exact form of $\mathbf{g}$ ). Finally $\boldsymbol{\eta}$ is a multiplicative gaussian noise with zero mean and standard deviation proportional (by a factor of 0.005) to the distance between the actual and desired end-effector positions, namely $\left|\mathbf{x}^{*}(t)-\mathbf{x}(t)\right|$. This noise factor is necessary to initiate the movement and to account for the trial-to-trial variability at the onset of movement (see the Appendix). 

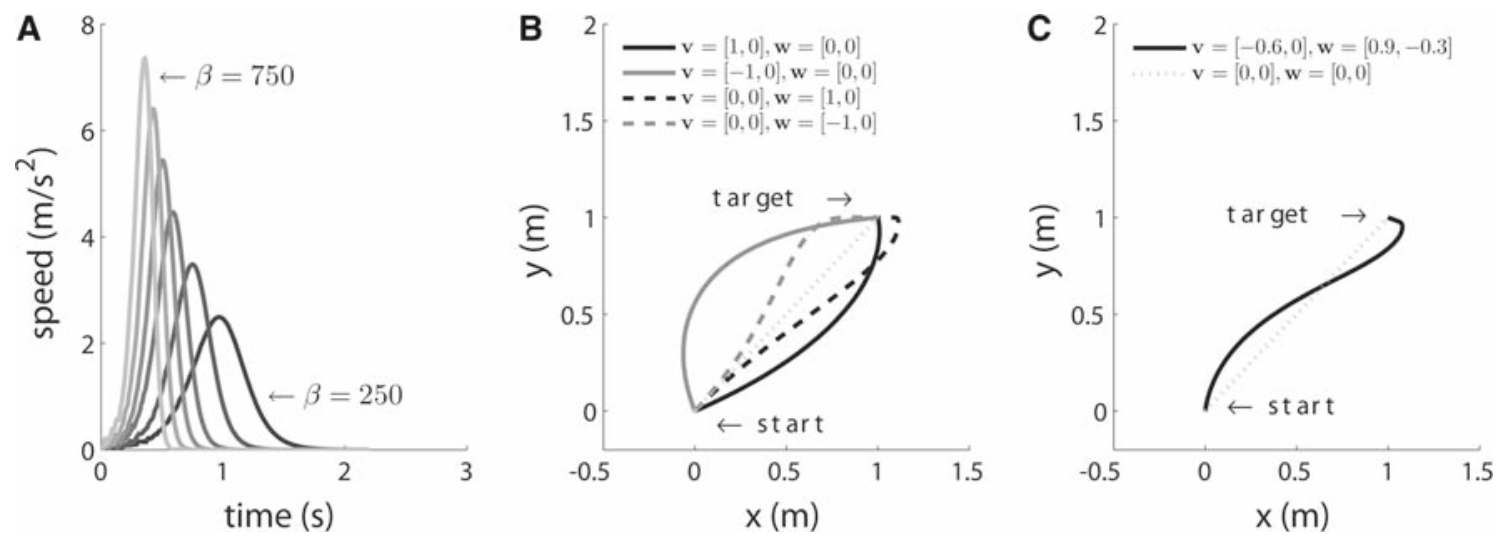

Fig. 3 a Effect of gradually increasing the parameter $\beta$ of the modified VITE model (see Eq. 1) on the velocity profile of the movement. Higher $\beta$ values increase the velocity peak and shorten the movement duration. b Behavior of the extended F2REACH model (see Eq. 2) under different repulsive forces $\mathbf{v}$ and $\mathbf{w}$, for illustrative purpose the forces shown are applied only on the horizontal dimension. The forces are modulated such that $\mathbf{v}$ affects mostly the beginning of the movement and $\mathbf{w}$ mostly the end of the movement. Note that the direction of the

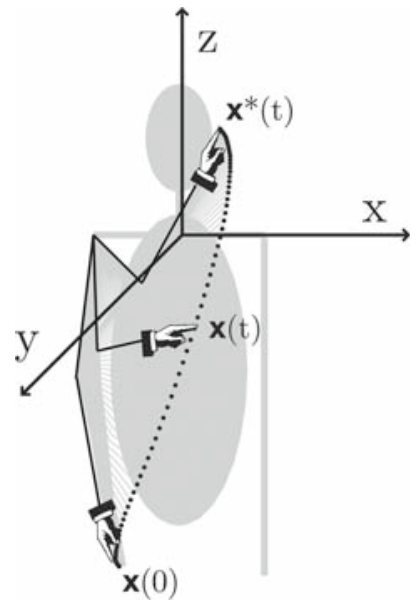

Fig. 4 Description of the task space. The hand position $\mathbf{x}(t)$ is represented in a 3-dimensional space centered on the chest, at the level of the shoulders. The input to the model consists of the initial hand position $\mathbf{x}(0)$ and final target position $\mathbf{x}^{*}(t)$

The above formulation makes two strong assumptions from a motor control point of view: (a) it takes as control signal the acceleration of the end-effector $\ddot{\mathbf{x}}$, expressed in an extrinsic 3-dimensional Euclidean space centered on the chest (see Fig. 4), and (b) it accounts only for a "high-level" control mechanism, in that it generates the desired end-effector kinematics, and does not account for the subsequent transformation required to control muscle activations.

Expressing the system in terms of desired acceleration is not constraining, since it is conceivable to assume that a neural population coding for the acceleration can be neurally integrated out to obtain a velocity control signal, which deviation from the straight trajectory is determined by the sign of the force. $\mathbf{c}$ By combining two forces $\mathbf{v}$ and $\mathbf{w}$ of different signs one can obtain very interesting deviations that change their direction during the execution of the movement. Reference values: $\alpha=50, \beta=500$, noise was set to 0.005 . Only the speed parameter $\beta$ is varied throughout the simulations, the two other parameters (time constant and noise) are fixed to the given values

can in turn be integrated out to have a position control signal, see Sauser and Billard (2006). Moreover, evidence that muscle activity may be governed by a kinematic signal, such as the acceleration, velocity or position, or any combination of these, has been found in the motor cortex (Wang et al. 2007). Note that we do not address the problem of redundancy mapping between desired hand kinematics and actual muscle activations in this paper. These assumptions will be further developed in Sect. 6.1.

The above system differs from the original VITE model in two ways (see the Appendix for the original VITE formulation). First, the dynamics of the system is now governed by a single second order differential equation and is thus expressed in terms of the end-effector acceleration. ${ }^{3}$ Second, we replaced the explicit time dependency of the original VITE system by introducing a bounded nonlinearity in the function $\mathbf{g}$. In the original VITE system, this explicit dependency in time through the priming signal let the velocity of the system grow exponentially in time, which created instabilities in the case of a long lasting perturbation, and was thus biologically implausible (your arm does not start accelerating if someone holds it).

\subsection{Extension of the original model: F2REACH model}

To account for the movement curvature, we next introduce a new functional $\mathbf{F}(\mathbf{x}(t))$ that corresponds to a virtual force

\footnotetext{
3 The original VITE system was driven by two coupled first-order differential equations. We reformulated this by writing the whole system as a second order differential equation. This allows us to relate explicitly the acceleration of the system to the force-field which we introduce in the following section.
} 
field, which encapsulates a geometrical representation of the task constraints. This force field is modulated by the dynamics of the control signal in order to preserve the bell-shaped velocity profile:

$$
\begin{aligned}
\ddot{\mathbf{x}}(t)= & -\alpha \dot{\mathbf{x}}(t)+\beta \mathbf{g}\left(\mathbf{x}^{*}(t)-\mathbf{x}(t)+\boldsymbol{\eta}\right) \\
& +\overbrace{\beta\left|\mathbf{g}\left(\mathbf{x}^{*}(t)-\mathbf{x}(t)+\eta\right)\right|}^{\text {modulation factor }} \overbrace{\mathbf{F}(\mathbf{x}(t))}^{\text {force field }}
\end{aligned}
$$

The force field $\mathbf{F}(\mathbf{x}(t))$ assigns a vector gradient to each position in space that expresses constraints related to: (a) objects in the environment that one needs to avoid (including the subject's body), (b) dynamic properties of the human body such as inertial properties of the limb, (c) extreme joint angles limits. The contribution of each of these constraints is simply summed to result in the virtual force field. The gradient of the force field at each point in space pushes the hand away from the undesired locations.

This force field framework reconciles the dynamic and kinematic aspects as well as intrinsic and extrinsic approaches to motor planning in a very convenient way. Instead of finding a compromise across systems that would operate simultaneously in conflicting coordinates (e.g., hand position and joint angles, see Sect. 2), our system provides both dynamic (acceleration) and kinematic (speed or position) control signals, taking into account (a) a target for the motion expressed in extrinsic kinematic coordinates and (b) intrinsic dynamic motion constraints. This reconciles the observation that objects in the environment such as a table may influence the kinematic planning of the movement ${ }^{4}$ (Brenner and Smeets 1995) and that knowledge of the arm dynamics is necessary for the kinematic planning of complex movements (Uno et al. 1989; Nakano et al. 1999; Sabes and Jordan 1997).

As the particular form taken by the force field is task and context dependent, we chose a very generic expression given by:

$\mathbf{F}(\mathbf{x}(t))=h(\mathbf{x}(t)) \mathbf{v}+(1-h(\mathbf{x}(t))) \mathbf{w}$

where $\mathbf{v}$ and $\mathbf{w}$ are constant force vectors that push the trajectory away from the straight-line generated by the rest of the system. $\mathbf{v}$ affects primarily the beginning of the movement, whereas $\mathbf{w}$ affects the end of movement (as illustrated in Fig. 3b, c). The modulation function $h$ that associates these two forces to different parts of the movement is given in the Appendix.

In our framework, a 3-dimensional reaching movement needs the specification of seven parameters in total: $\beta$ that controls the amplitude of the velocity's peak and two 3-dimensional repulsive forces $\mathbf{v}$ and $\mathbf{w}$, where the time con-

\footnotetext{
4 This type of computation is natural (and especially useful) if the movement is considered in a constantly varying environment full of external objects, instead of isolated in an artificial experimental setup.
}

stant $\alpha$ and noise can be fixed to 50 and 0.005 respectively. We will show next that the latter two forces give a crude representation of the volume and geometry of the body around which the hand must navigate.

To conclude the description, control policies of the form of autonomous differential equations such as the one proposed here are particularly interesting, as they allow online modifications of the input variables. Thus a very nice property of our model is its robustness to external perturbations, where the model shows smooth adaptation to changes such as blocking or displacing the arm and displacing the target (simulation results not shown here).

\section{Experiments}

\subsection{Subjects}

Ten healthy subjects, five female and five male of mean age $33 \pm 11$ years volunteered for the study. All the participants except for two were right-handed according to the Edinburgh handedness test (Oldfield 1971). All the subjects were naive as to the purpose of the study and had no history of neurological or musculoskeletal deficits.

\subsection{Procedure}

The subjects were asked to perform natural reaching movements toward targets situated on their head. In order to obtain entirely natural and fully unconstrained movements, the target positions were specified verbally (for example we gave instructions such as "on the go signal touch your nose"). The subjects were left free to determine the location of the reaching target (e.g., at the tip of the nose or just above it), but they were instructed to reach to exactly the same location across one block of repetitions of the same movement. There were six target positions, shown in Fig. 5a, indexed as follows: (1) nose, (2) right ear, (3) left ear, (4) top of the head, (5) under the chin and (6) back of the head. Given that the subjects had different arm lengths and given that the targets were defined with respect to the subject's head, the length of the hand path varied importantly across subjects and movements. This was done on purpose to test the ability of the model to reproduce the generic characteristics of the movements and to account for such body variabilities, which we consider task-independent. The subjects were standing in order to limit undesirable movements of the upper body. There were no external constraints that would confine the movement range. The movements were performed with the right hand independently of the handedness of the subject, since handedness was shown not to affect spontaneous self-oriented movements (Dalby et al. 1980; Lavergne and Kimura 1987). In order to verify the gener- 

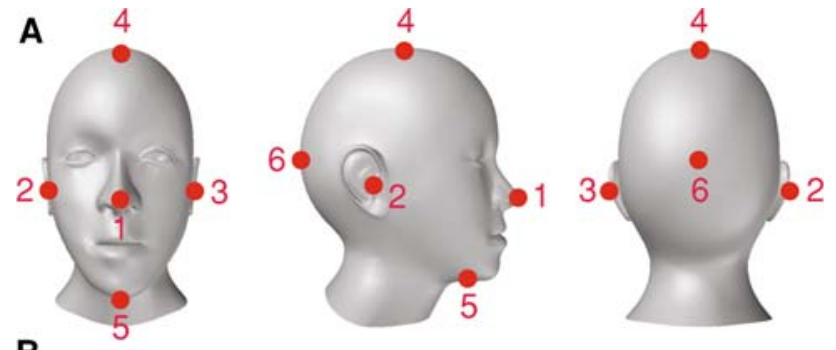

B
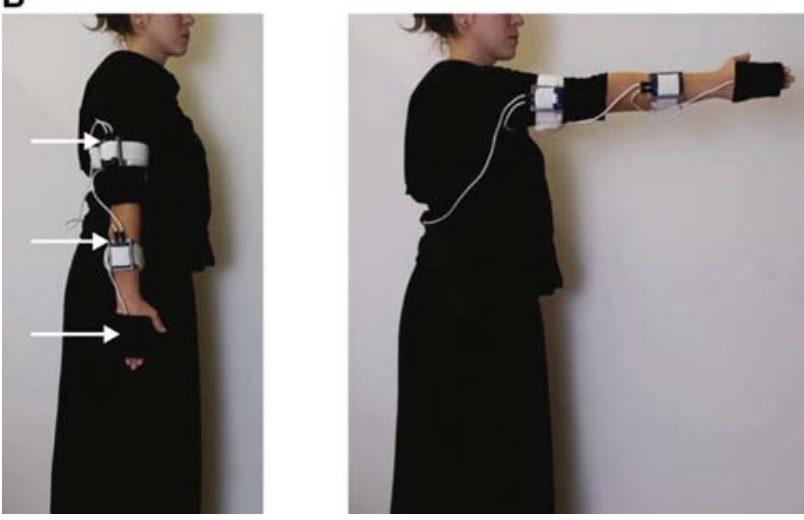

Fig. 5 a Target positions on the head used in our experiment. b Two initial conditions that yield both highly and gently curved movements. The three motion sensors are indicated with arrows

alization of our model over movements with different curvature levels, movements were initiated from two different locations, shown in Fig. 5b: (1) upright position with the arm extended along the body that yielded highly curved movements and (2) upright position with the arm extended in front of the body that yielded gently curved movements. Prior to each experiment, the subjects were asked to assume the same starting position, which was verified by the experimenter. The subjects had at least one trial of practice per movement to ensure that they had understood the instructions. Each movement was repeated five times in order to have a measure of its inherent variability and consequently a measure of the precision of the model's reproduction.

\subsection{Data acquisition}

Data was recorded using 3D inertial measurement units/ motion sensors (Xsens Technologies B.V., The Netherlands). The sensors were attached on three arm segments (the upper arm, the forearm and the hand) and were calibrated in the upright position with the arm vertical (see Fig. 5b, left). The orientation of the three arm segments during the execution of the movements was recorded at a frequency of $50 \mathrm{~Hz}$.

\subsection{Data analysis}

All analyses were performed with custom software written in Matlab (Mathworks, Natick, MA, USA). The trajectories of each arm segment were reconstructed using the orientation matrices recorded by the inertial measurement motion sensors. We used only unfiltered raw values. The movements of interest were extracted using criteria such as percentage of velocity change. The samples were aligned in time so that the inter-trial Euclidean distance per movement and subject (five samples) is minimal. The movement mean and standard deviation (SD) of each trajectory for each movement type and for each subject was computed with respect to the aligned signals. We then solved numerically the original VITE and extended F2REACH models for each of the mean movements, with a time step of $20 \mathrm{~ms}$. The models' parameters were fixed using $3^{3}$ and $3^{7}$ factorial experimental designs respectively, coupled with a local search procedure (Neter et al. 1996; Hoos and Stützle 2004).

To evaluate the predictions of the two models we measured the following Euclidean distances and deviation indices: ${ }^{5}$ (1) mean deviation (MD) of the predicted hand trajectory compared to the measured hand trajectory at each point in time, (2) mean squared error (MSE), (3) hand trajectory deviation index (HTDI) defined as the ratio between the maximal distance across the modeled $\mathbf{x}^{m}(t)$ and real $\mathbf{x}^{r}(t)$ mean trajectories over the total length of the real path,

$$
\mathrm{HTDI}=\frac{\max _{i=1, \ldots, N}\left|\mathbf{x}^{m}(i)-\mathbf{x}^{r}(i)\right|}{\sum_{i=1}^{N-1}\left|\mathbf{x}^{r}(i+1)-\mathbf{x}^{r}(i)\right|}
$$

where $N$ is the number of points sampled (see Fig. 6a), (4) speed deviation index (SDI) and finally (5) total acceleration deviation index (ADI), both defined in Fig. 6b. We also considered the standard deviation trajectory (SD) as a possible limit prediction (see Fig. 6c for a definition). We further assessed the curvature index of recorded and modeled movements, defined as the ratio between the total arc length of the hand path and the Euclidean distance that separates the initial and final positions. For example a curvature index of 1 indicates a perfectly straight path and a curvature of $\pi / 2$ corresponds to a semicircular path. Finally, the speed asymmetry index was defined as the ratio $\left(S^{a}-S^{d}\right) /\left(S^{a}+S^{d}\right)$ where $S^{a}$ is the distance traveled up to the time at which the velocity is maximal (referred to as the acceleration phase) and $S^{d}$ the distance traveled from the time at which the velocity is maximal until the end of the movement (deceleration phase). An additional measure of the precision of the original VITE and extended F2REACH models is the percentage of trajectory points predicted by the models that are comprised within the volumes defined by 1 and 2 SD away from the recorded mean trajectory (per subject and movement type, established over five repetitions of the movement, see Fig. $6 \mathrm{c}$ ). This measure accounts for the variability inherent to goal-directed reaching

\footnotetext{
5 The deviation indices are adapted from Nakano et al. (1999) and Biess et al. (2007).
} 

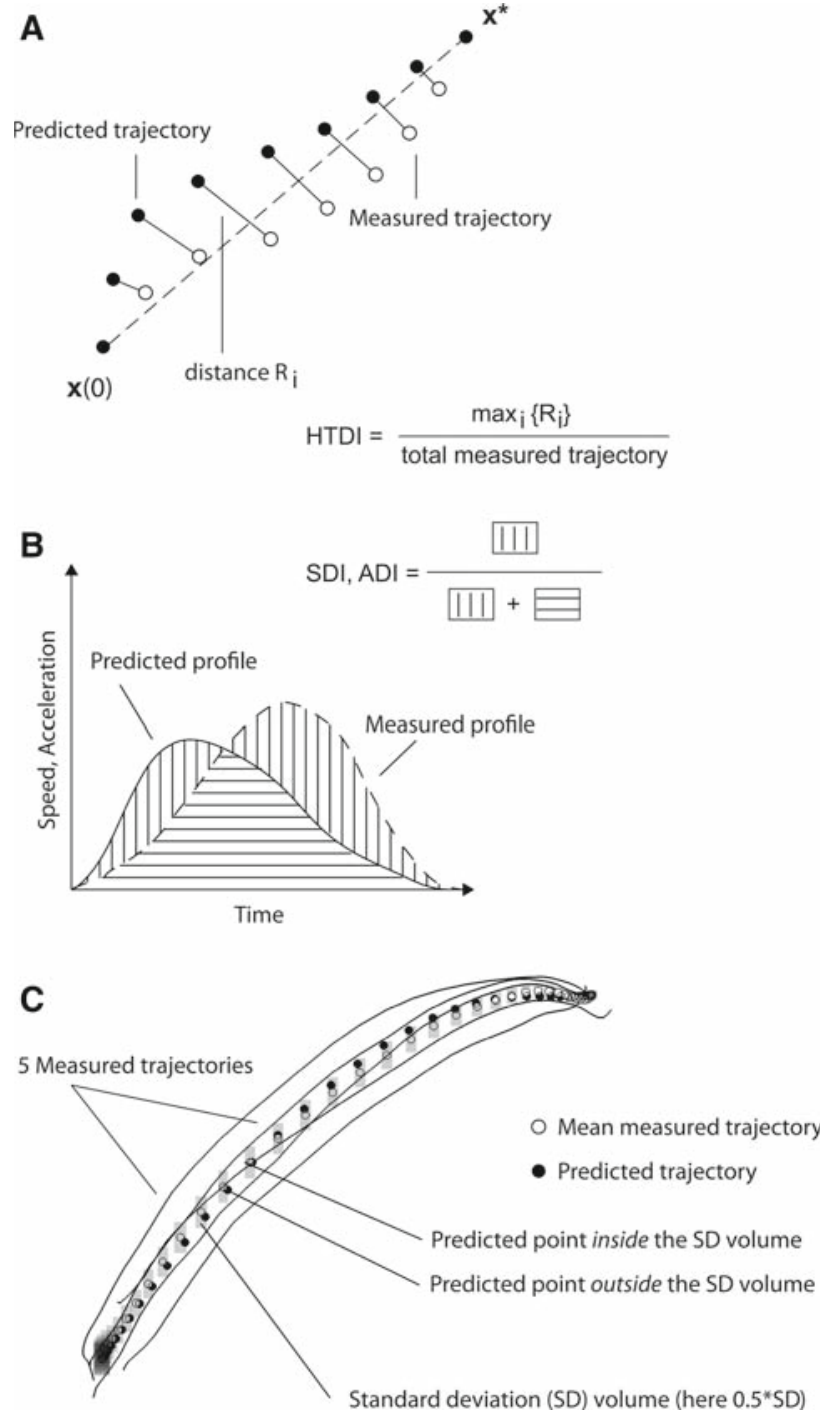

Fig. 6 Definitions of error measures. a The hand trajectory deviation index $(H D T I)$ of measured and predicted hand trajectories is the ratio of the maximum distance, $R=\max _{i=1, \ldots, N} R_{i}$, between the two trajectories matched in time over the total length of the measured path. b The speed deviation index ( $S D I)$ and total acceleration deviation index $(A D I)$ are defined as the ratio of the noncommon area enclosed by the measured and predicted speed/acceleration profiles and the total enclosed area. c Standard deviation volumes (SD), comprised within a multiple of the standard deviation distance (computed from the mean trajectory of five movement trials per subject and movement type) at every point of the movement trajectory. A SD trajectory would follow the corresponding corners of these volumes. We consider that a point was well predicted if it is contained inside the SD volume of its measured counterpart, thus enforcing a higher precision at points with very low variability

movements (Harris and Wolpert 1998; Todorov and Jordan 2002) and penalizes imprecision in parts where the variance of the movement is minimal. For example, the subjects were more consistent in the vicinity of the initial and target positions.

\section{Results}

In this section we report on a systematic assessment of how well the original VITE and our extended F2REACH models account for the kinematics of the recorded human movements. We also discuss the biological plausibility of our model's parameters. Finally, we conduct a stability analysis of the F2REACH model and define conditions under which the target is a stable attractor of the model and therefore guaranteed to be reached.

\subsection{Observed data statistics}

We first assessed the general characteristics of the recorded movements (summarized in Table 1). The movements addressed had large spatial extent (mean path length of $1.23 \mathrm{~m}$ ) with significantly longer path lengths in the first experimental condition (see Fig. 5b) when compared to the second experimental condition (mean path lengths of 1.7 and $0.95 \mathrm{~m}$ respectively). Movements in the first condition lasted longer with mean durations of 1.3 and $1 \mathrm{~s}$, respectively. Most importantly, the movements in the first condition were significantly more curved with a mean curvature index of 1.59 compared to 1.21 in the second condition. In addition, the curvature indices of the recorded movements were distributed homogeneously between quasi-straight $(<1.1)$ and highly curved $(>2)$.

We expected to see substantial trial-to-trial fluctuations due to noise of the motor system (Todorov and Jordan 2002), which motivated us to model the mean trajectory of the movement rather than the separate trials. We believe that the mean movement captures the intrinsic nature of the movement, which is task-relevant and free of noise. An example of the inherent variability across trials per subject and movement type is shown in Fig. 7a. Figure 7b shows that the intersubject variability (attributed to the difference in embodiment of the subjects) is much more important.

\subsection{Comparison between the observed and modeled data}

Here we assess how well the original and extended models reproduce the human data. The mean movement trajectories were simulated with both the original VITE and our extended F2REACH models. Typical examples of measured and predicted hand path trajectories are given in Fig. 8. The first row in each example shows the five hand trajectories of the movement projected in the $x y$-, $x z$ - and $y z$-planes relative to a schematized humanoid. The second row shows the projections of the mean recorded trajectory and generated model trajectories. The subject's trials are represented with light grey lines and show the inherent variability of the movement. The third row shows the $x$-, $y$ - and $z$-components of the hand trajectories with respect to time in order to show the quality of the model predictions at the temporal level. 
Table 1 Path length, duration and curvature index of the movements in the two experimental conditions (see Fig. 5b)

\begin{tabular}{lllll}
\hline & Condition 1 & Condition 2 & 2 Conditions & 10 Subjects \\
\cline { 3 - 5 } & & & $P$ value & NS \\
\hline Path length (m) & $1.70 \pm 0.32$ & $0.95 \pm 0.18$ & $<0.001$ & $<0.001$ \\
Duration (s) & $1.28 \pm 0.26$ & $0.97 \pm 0.20$ & $<0.001$ & $<0.001$ \\
Curvature index & $1.59 \pm 0.22$ & $1.21 \pm 0.11$ & NS \\
\hline
\end{tabular}

We also give one-way ANOVA results for the initial condition and subject effects on these variables. The movements in condition 1 were significantly longer in time and space and significantly more curved when compared to the movements in condition 2 . The recorded movements differed significantly across subjects only in their duration

Table 2 Mean deviation (MD), mean squared error (MSE) and mean deviation indices (see Fig. 6) for the trajectory (HTDI), speed (SDI) and acceleration (ADI) ( \pm standard deviation) of the hand as predicted by the extended F2REACH and original VITE models

\begin{tabular}{lccr}
\hline & F2REACH model & SD & VITE model \\
\hline MD $(\mathrm{mm})$ & $18.85 \pm 8.10$ & $35.67 \pm 11.63$ & $132 \pm 71$ \\
MSE $\left(\mathrm{cm}^{2}\right)$ & $5.62 \pm 5.34$ & $15.93 \pm 10.61$ & $431 \pm 413$ \\
HTDI & $0.031 \pm 0.010$ & $0.04 \pm 0.02$ & $0.25 \pm 0.06$ \\
SDI & $0.11 \pm 0.03$ & $0.50 \pm 0.11$ & $0.29 \pm 0.12$ \\
ADI & $0.38 \pm 0.07$ & $0.60 \pm 0.08$ & $0.51 \pm 0.13$ \\
\hline
\end{tabular}

We also consider the trajectory comprised within one standard deviation (SD) from the mean trajectory (per subject and movement type, computed as described in Fig. 6c) as an indication for the limit prediction that would be acceptable for a model. This SD trajectory represents the inherent variability of the movement. One-way ANOVAs performed on the error measures of the extended F2REACH model show that the effect of the subject performing the movement was not significant and that the movements in the second initial condition, i.e., movements with lower curvature, tended to be slightly better predicted (MD and MSE only)
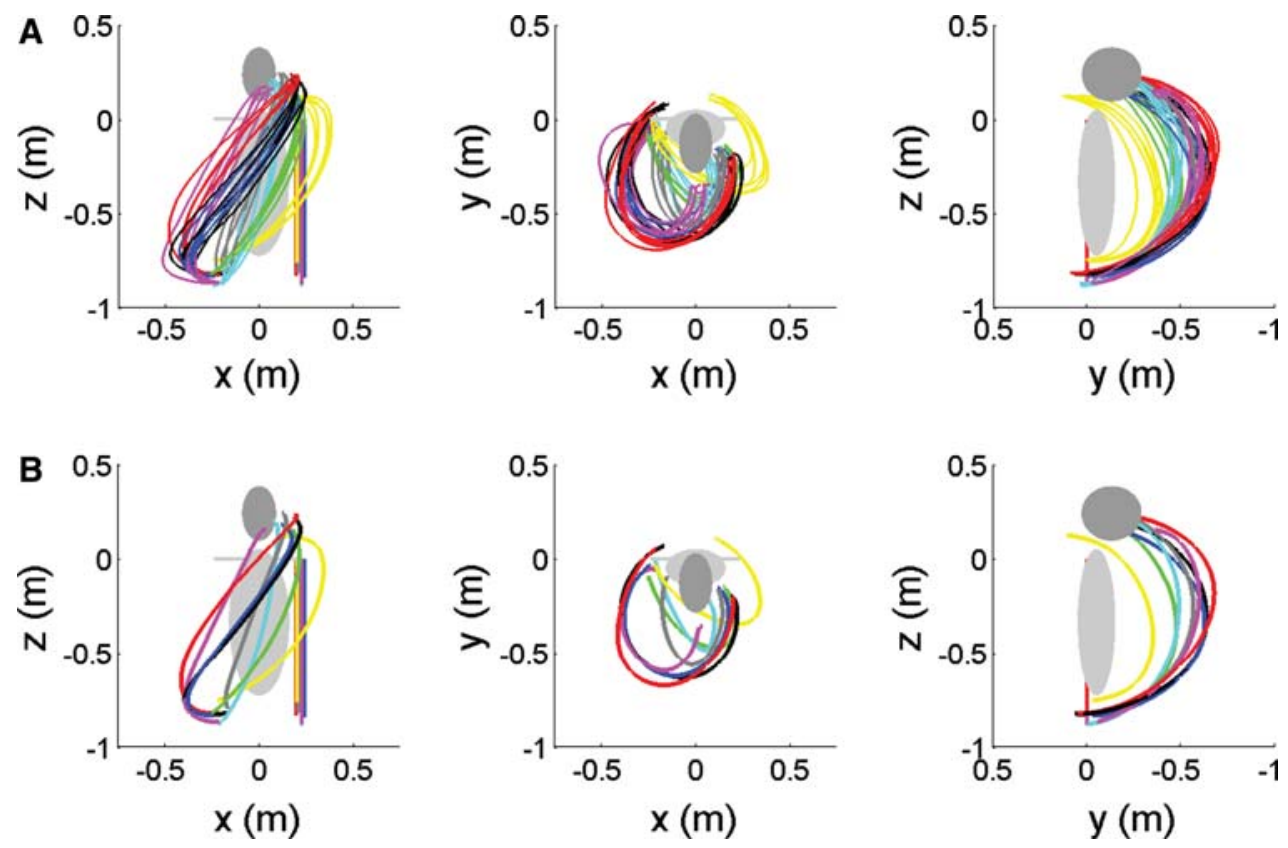

Fig. 7 Trajectories of the hand for ten subjects performing five repetitions of the same movement, reaching to the left ear (movement 3) with the right arm in condition 1 (see Fig. 5). The hand trajectories are shown relative to a schematized humanoid and the color refers to the same subject. a All the movement trajectories are shown in order to emphasize the movement's inherent variability. Note that this intra-

subject variability is lower than the inter-subject variability, i.e., the hand trajectories of one subject are consistent when compared to those of the other subjects. b Only the mean movements are shown. The inter-subject variability can be partially attributed to differences in the subjects' arm lengths and shoulder positions (see color-coded arms) 
I ?

A

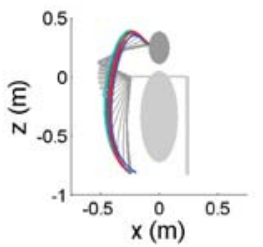

B

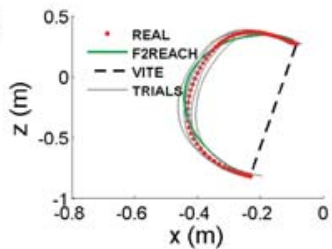

C

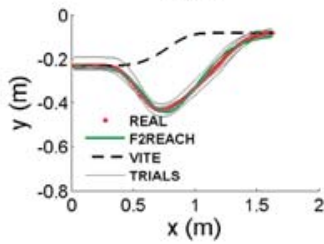

II

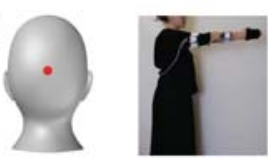

A

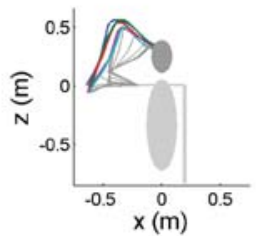

B

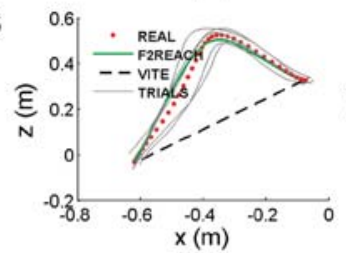

C

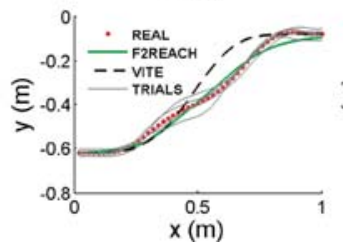

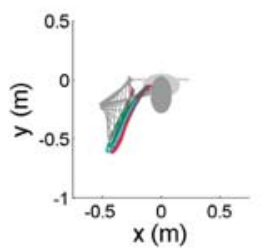
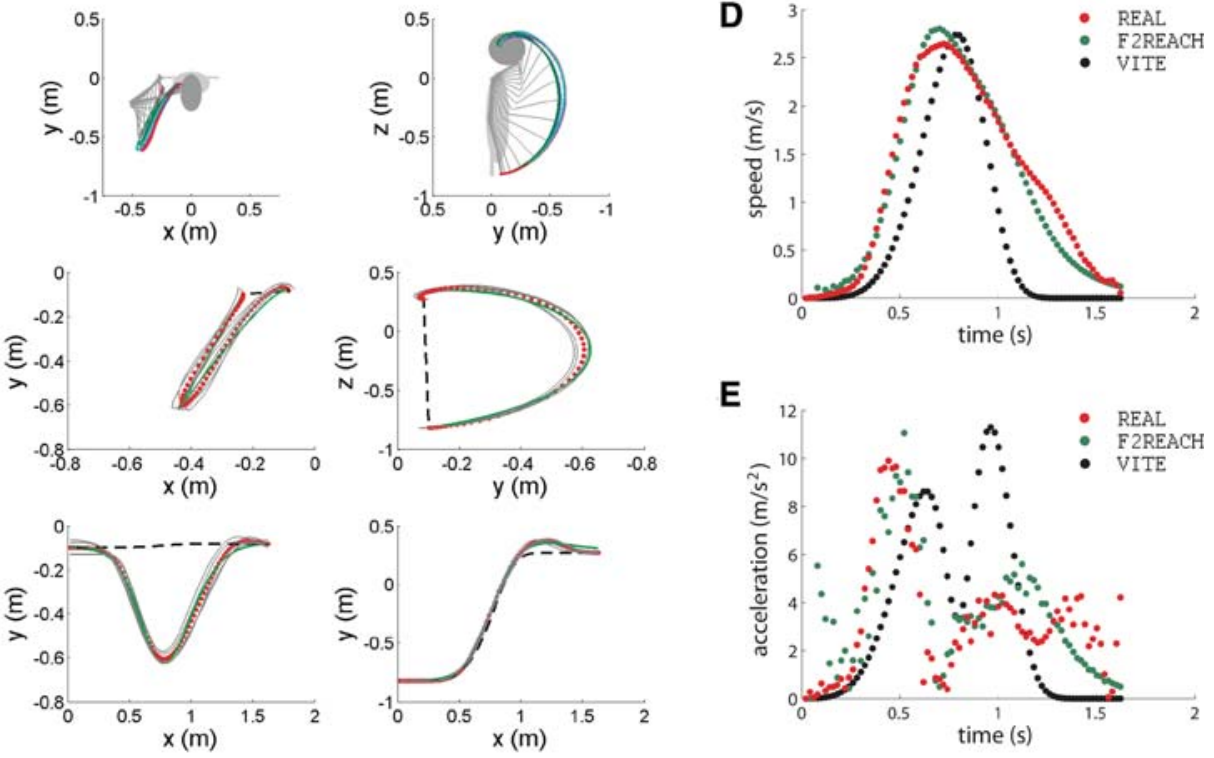

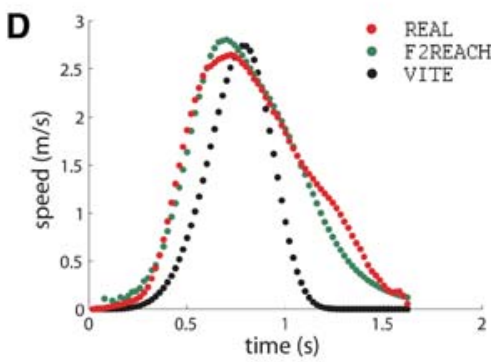

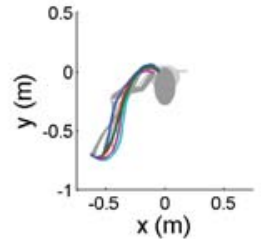
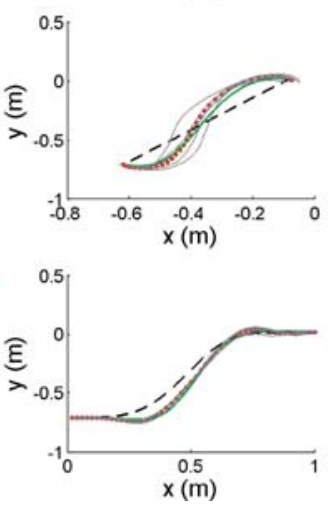
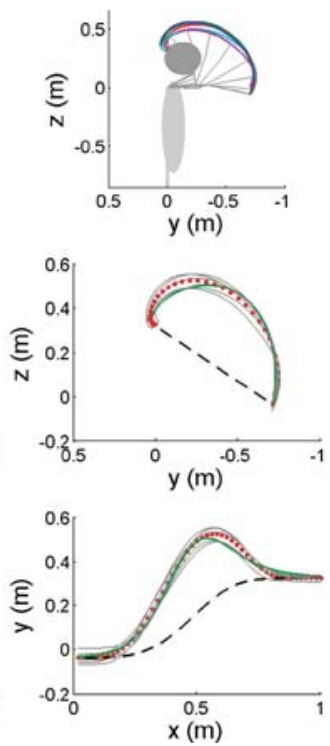

D
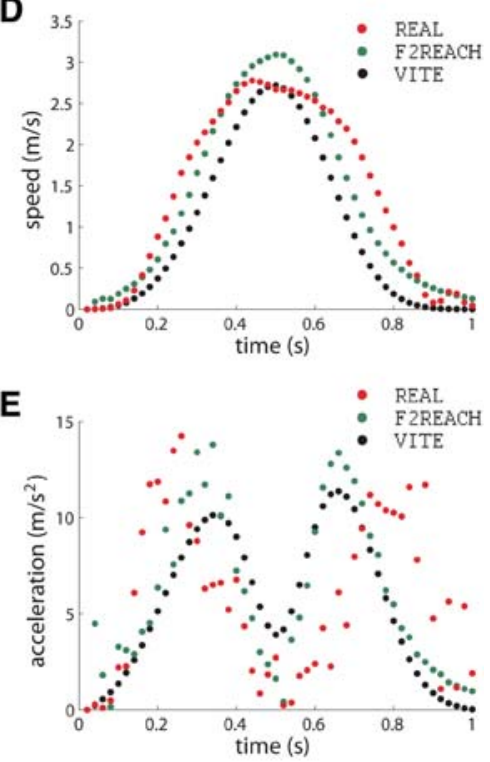

Fig. 8 Two examples of typical movements. The recorded human data is shown with points that respect the sampling rate, the original VITE model is shown with a dashed line and our extended F2REACH model with a plain line. $I$, The subject reaches for the back of the head (movement 6) with as initial condition the right arm extended along the body (condition 1). II The subject reaches for the back of the head (movement 6) with as initial condition the right arm extended in front of the body (condition 2). a The five recorded hand trajectories of the move- ment projected in the $x y-, x z$ - and $y z$-planes and shown relative to a schematized humanoid. b The measured and predicted mean movement trajectories projected in the $x y$-, $x z$ - and $y z$-planes. The light grey trajectories are the five trials and reflect the intra-subject variability per movement type. $\mathbf{c}$ The $x$-, $y$ - and $z$-components of the measured and predicted mean movement trajectories shown with respect to time. d The measured and predicted speed profiles of the movement. e The measured and predicted total acceleration profiles of the movement 
Table 3 Measured (M) velocity peak amplitude and peak time, asymmetry and curvature indices ( \pm standard deviation) compared to those predicted by the extended (F2REACH) and original (VITE) models

\begin{tabular}{lccccc}
\hline & Measured (M) & $\begin{array}{l}\text { F2REACH } \\
\text { model }\end{array}$ & $\begin{array}{l}\text { M versus F2REACH } \\
P \text { value }\end{array}$ & $\begin{array}{l}\text { VITE } \\
\text { model }\end{array}$ & $\begin{array}{l}\text { M versus VITE } \\
P \text { value }\end{array}$ \\
\hline Velocity peak amplitude (m/s) & $2.26 \pm 0.68$ & $2.37 \pm 0.69$ & $<0.05$ & $8.22 \pm 59.31$ & NS \\
Velocity peak time (s) & $0.50 \pm 0.14$ & $0.53 \pm 0.13$ & NS & $0.59 \pm 0.19$ & $<0.001$ \\
Asymmetry index & $-0.08 \pm 0.15$ & $-0.10 \pm 0.11$ & NS & $0.03 \pm 0.06$ & $<0.001$ \\
Curvature index & $1.40 \pm 0.26$ & $1.36 \pm 0.23$ & NS & $1.03 \pm 0.22$ & $<0.001$ \\
\hline
\end{tabular}

There were no significant differences between the measured and extended model variables (with the exception of a small difference in the velocity peak amplitude), whereas significant differences were found between the measured and original model for three of the four variables addressed (all except for the velocity peak amplitude)

Finally, on the right we show the measured and predicted speed and acceleration profiles. One can see that, unlike the original VITE model, the F2REACH model is generally in very good agreement with the human data.

We systematically evaluated the predictions of the original VITE and extended F2REACH with several Euclidian distances and deviation indices defined in Sect. 4.4. The results are summarized in Table 2 and show that our model is highly precise at reproducing the kinematics of the recorded movements. The deviation indices are much smaller, generally on a different order of magnitude than those from the SD trajectory and always smaller than the original VITE model. The mean deviation was less than $2 \mathrm{~cm}$ for movements of average path length superior to $1 \mathrm{~m}$.

We performed one-way ANOVAs for the extended model using, as dependent data, the different error measures defined in the preceding paragraph. The results show that, regardless of the error measures used, we did not find an effect of the subject executing the movements $(P>0.05$, with the exception of two subjects for the HTDI and ADI deviation indices). This indicates that our model performed equally well across the ten subjects. A significant effect $(P<0.001)$ was observed for the two experimental conditions (see Fig. 5) for the mean deviation (MD), mean square error (MSE) and speed deviation index (SDI) suggesting that the model is better at predicting low rather than high curvatures. This result is not very surprising since the force field in our model is parameterized with two constant forces, thus approximating the real force field underlying the movement. The more a movement is curved, the more imprecisions related to this parametrization affect the model's performance. Finally, the original and extended models differed significantly in their predictions for all the error measures $(P<0.001)$.

We have further investigated whether our model captures the major temporal characteristics of the movement. We compared the VITE and F2REACH models' predictions to the real data for the peak amplitude, time at peak amplitude and speed asymmetry index, see Table 3. One way ANOVAs confirmed a very good match between our model's prediction and the data for all the above quantities (except for the velocity peak which was slightly lower, $P<0.05$ ), whereas the predictions of the original VITE model differed significantly from the data $(P<0.001)$ except for the velocity peak amplitude. To illustrate the quality of the extended and original VITE models' predictions for the time-dependency of the signals, in Fig. 9 we compare instances of measured and predicted speed profiles (normalized in time). Finally we looked at the percentages of trajectory points comprised within the volumes defined by one and two standard deviations (SD) in order to evaluate the performance of the models at portions where the movement is very precise and systematic over trials (see Sect. 4.4 for details). The results show that $81 \%$ of the hand trajectory points predicted by our model were within two SDs of the mean trajectory against $40 \%$ of the points predicted by the original VITE model (Table 4 shows also the result for 1D).

One should emphasize that the F2REACH model generates these 3-dimensional movements using few parameters: $\beta$ that controls the amplitude of the velocity and the two repulsive force vectors $\mathbf{v}$ and $\mathbf{w}$ (see Fig. $3 \mathrm{~b}$ ) that parameterize the force field surrounding the subject. The other two parameters $\alpha$ (time constant) and noise were fixed to 50 and 0.005 in all the simulations. The high accuracy with which the model manages to replicate the movements confirms that the model encapsulates the important features underlying free reaching movements. The force field is a key variable of the model. Next we show that the force field can be interpreted in relation to the bio-mechanical constraints of the subject's body.

\subsection{Understanding the force field}

Figure 10 shows the components of the virtual repulsive forces $\mathbf{v}$ and $\mathbf{w}$ parameterizing the force field of the F2REACH model (Eq. 2). We observe that the values of the components are clustered in two groups depending on the starting location of the movement. They are, thus, consistent 


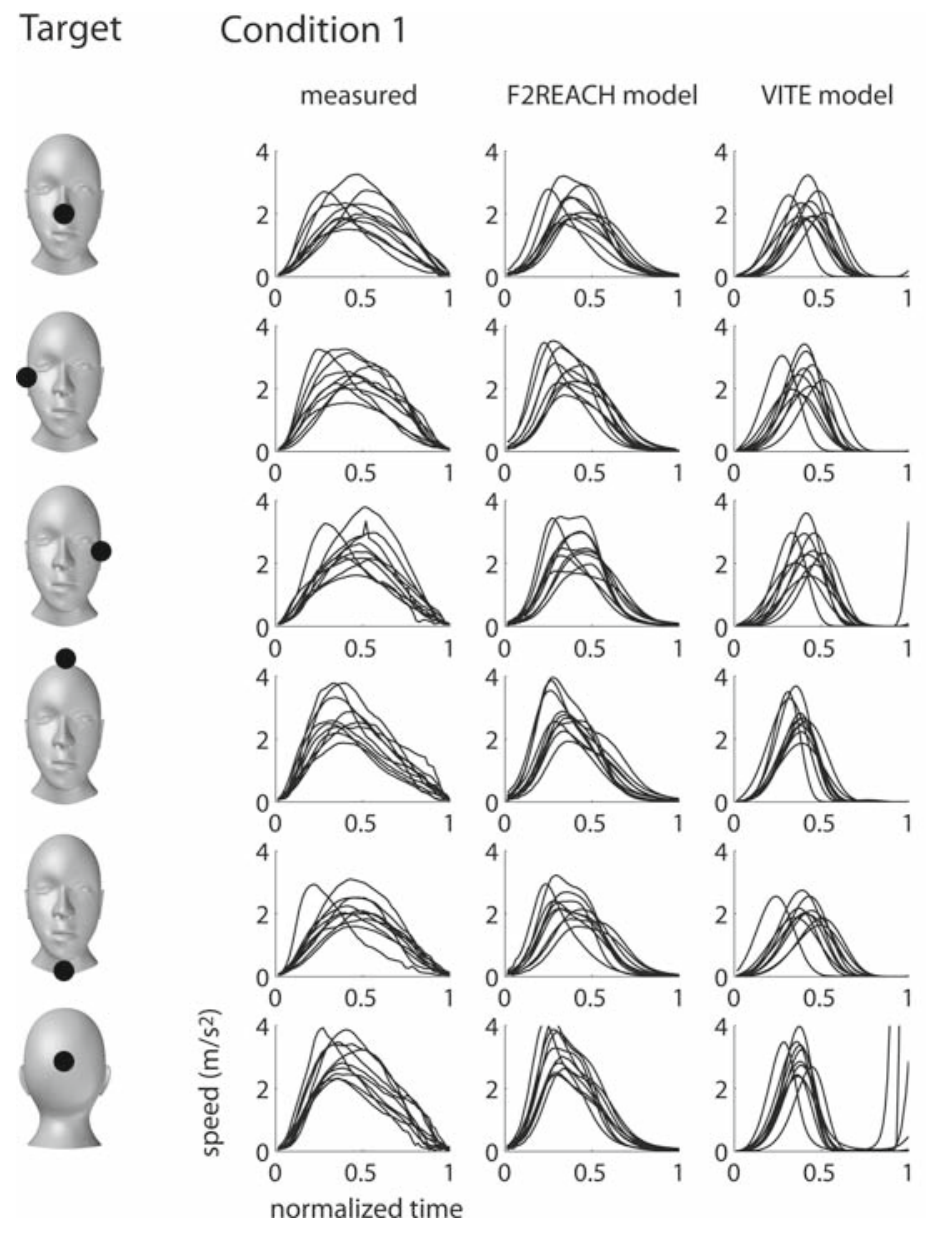

\section{Condition 2}

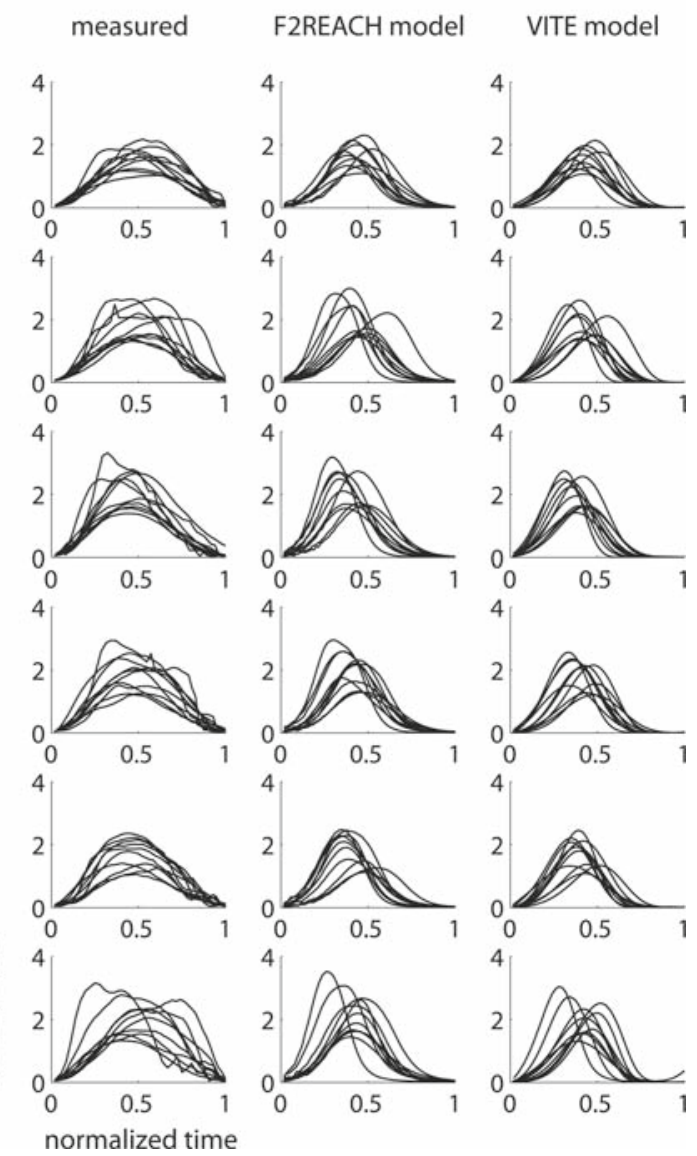

Fig. 9 Normalized time speed profiles of the measured human data and as predicted by the extended F2REACH and original VITE models, for the six target positions and the two initial conditions (see Fig. 5)

within the same condition (see Fig. 10a). The fact that movements to different targets are also clustered (forces underlying similar movements have similar components) suggests a certain regularity in the force field (see Fig. 10b). Finally, the trials related to one movement are clustered according to the subject executing the movement, which shows once more that the parameter values found for the repulsive force fields are not arbitrary (see Fig. 10c). Recall that the sign of the force vector governs the direction of the deviation and that, according to the expression of the modulating function $h$, the resulting force $\mathbf{F}(\mathbf{x}(t))$ coincides with $\mathbf{v}$ at the beginning of the movement and with $\mathbf{w}$ at the end of the movement, $\mathbf{F}(t=0)=\mathbf{v}$ and $\mathbf{F}\left(\mathbf{x}=\mathbf{x}^{*}\right)=\mathbf{w}$.

Closer analysis of the clusters shows that the force $\mathbf{v}$, dominating the beginning of the movement, is highly dependent on the starting location in the $x$ and $y$ coordinates (see Fig. 4), whereas the force $\mathbf{w}$, dominating the end of the movement, varies according to the $z$ direction. An intuitive explanation for this result is shown in Fig. 11 where we show the direction and amplitude of the repulsive forces $\mathbf{v}, \mathbf{w}$ and their modu-
Table 4 Percentages of predicted trajectory points comprised within one and two standard deviation volumes (1 SD and 2 SD), see Fig. 6c, for the extended F2REACH and original VITE models

\begin{tabular}{lll}
\hline & F2REACH & VITE \\
\hline $1 S D$ & $54.64 \pm 19.17$ & $31.11 \pm 8.44$ \\
2 SD & $80.89 \pm 14.82$ & $39.76 \pm 9.18$ \\
\hline
\end{tabular}

This error measure is highly restrictive as it penalizes the model predictions at points where the five trials per subject and movement type are very consistent

lated sum $\mathbf{F}(\mathbf{x}(t))$ (Eq. 3) for different types of movement. We see that, in the second starting position (arm extended in front of the body) the subject pushes his or her hand in the direction of the target (see Fig. 11a), whereas, in the first starting position (arm extended along the body) the subject must first push the hand to the right in order to avoid the body, and then bring the hand downwards in order to avoid reaching the limit of the shoulder joints (see Fig. 11b). 
Fig. 10 Components of the repulsive forces $\mathbf{v}$ and $\mathbf{w}$. a We show the components of the first repulsive force $\mathbf{v}$ for the two conditions: arm extended along the body (red) and arm extended in front of the body (blue). Two practically non-overlapping clusters can be observed parameter values within one condition. b We show the components of the second repulsive force $\mathbf{w}$ in the first condition for the six targets (different scale). Again the parameter values are clustered such that movements oriented toward one target are close together, showing a regularity in the repulsive force field. $\mathbf{c} \mathrm{We}$ show the components of the first condition and target right ear for the ten subjects. Clusters corresponding to the subjects can be identified for the five trials representing the movement showing a consistency of the repulsive force $\mathbf{v}$ in the first
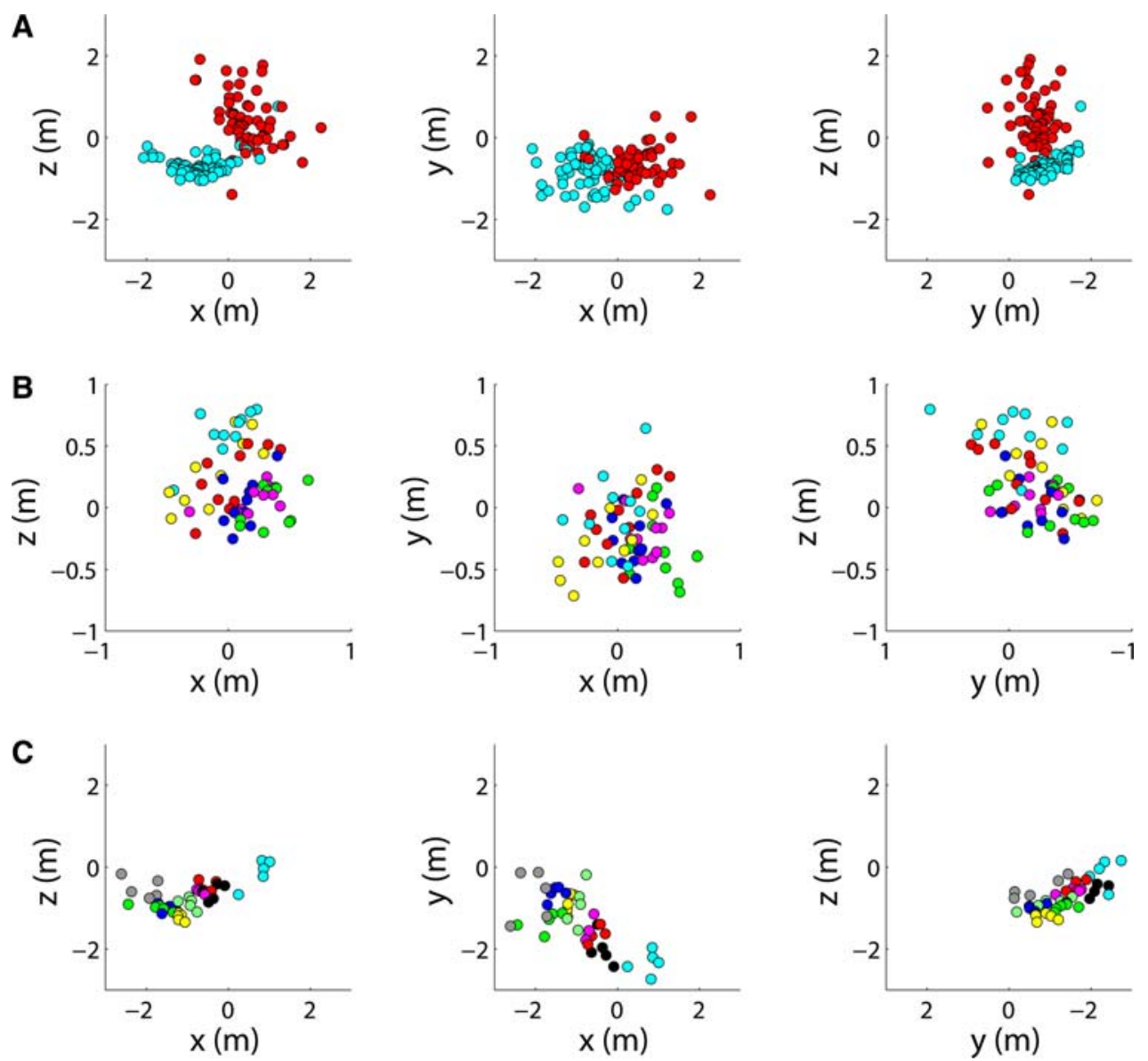

\subsection{Stability analysis of the model}

ing from the same initial condition, we compared the values found for the force components when reaching to two different targets (Fig. 11b, c). Unsurprisingly, the repulsive vector $\mathbf{v}$ is coherent across conditions irrespective of the target position, whereas the repulsive vector $\mathbf{w}$ depends on the target position and moves along the normal to the head surface at the target's position.

We also considered whether the magnitude of the repulsive force is related to the geometry of the subject's body, such as the length of the forearm for example. We observed a linear correlation between these two quantities (shown in Fig. 12): the shorter the arm, the more the hand must be pushed away to circumvent the head. Finally, we observed that the vectors of repulsive forces were coherent across subjects. These results are in agreement with the driving hypothesis of our model, namely that the curvature of reaching movements is the result of an explicit encapsulation of the task constraints in a control system which would, in the absence of constraints, produce straight-line motions. However, the opposite is not true, as we find non-null forces for quasi-straight movements, which are parallel to the motion. In the movements we have considered here, the task constraints comprise geometrical constraints related to the body.
The dynamical system described in Eq. 1 is globally asymptotically stable around a unique equilibrium point, the target position $\mathbf{x}^{*}$. We have omitted the analytical proof but the interested reader can convince themselves by computing the determinant of the Jacobian of the dynamical system around the fixed point and observe the latter to be always negative. Next we define the conditions under which the F2REACH model including the repulsive force field (see Eq. 2) is guaranteed to converge to the target. Let there be a perturbation that drifts the hand far away from the initial and target positions, such that $|\mathbf{x}(t)-\mathbf{x}(0)|-\left|\mathbf{x}(t)-\mathbf{x}^{*}(t)\right|<\varepsilon$, with $\varepsilon \in \mathbb{R}$ very small and $h(\mathbf{x}(t))$ and $1-h(\mathbf{x}(t))$ approaching $1 / 2$. The system converges to a stable state iff $|1 / 2(\mathbf{v}+\mathbf{w})|<1$ such that the amplitude of the repulsive force field is smaller than the normalized attracting vector, i.e., the distance separating the target from the present position only gets smaller through time. ${ }^{6}$ Note that all the forces' components found in our study satisfied the above condition.

\footnotetext{
6 This result is, however, not valid in the vicinity of the initial position, which acts a second unstable attractor. Since this affects only a transient part of the motion (onset of the movement), which is unlikely to undergo perturbations, this could be ignored for the present study.
} 

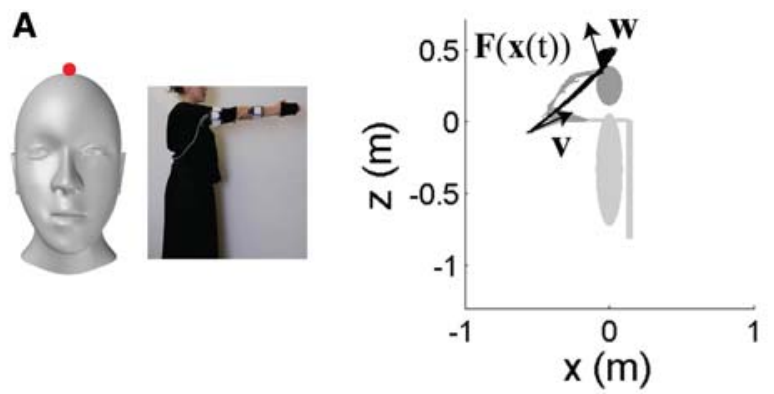

B
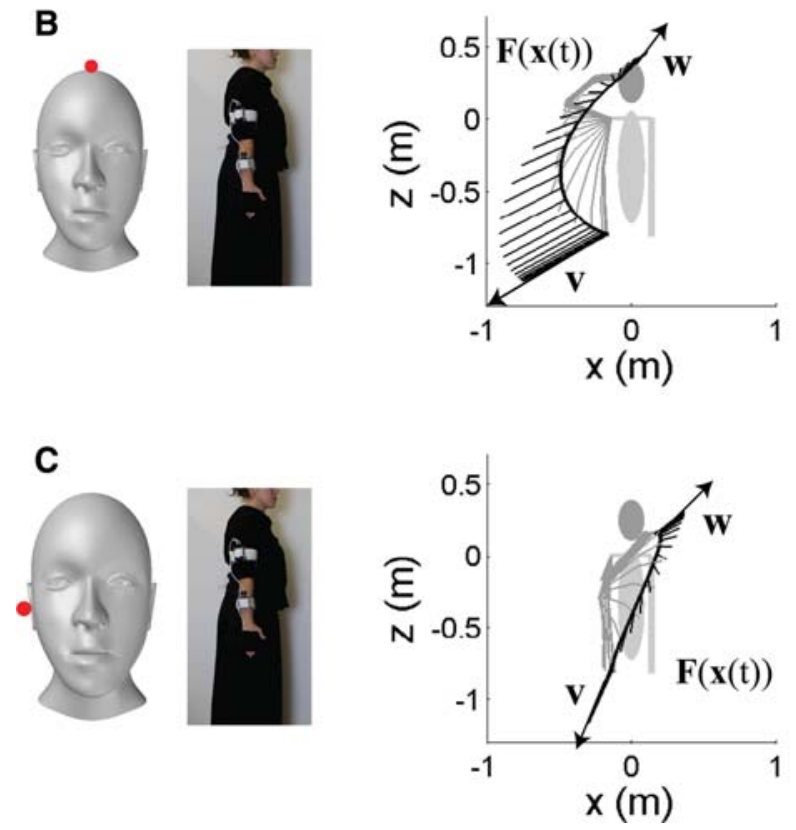

Fig. 11 Physical interpretation of the directions and amplitudes found for the repulsive forces $\mathbf{v}, \mathbf{w}$ and their modulated $\operatorname{sum} \mathbf{F}(\mathbf{x}(t))$ in our extended F2REACH model (Eqs. 2 and 3) relative to a schematized humanoid. Three movements of the same subject are shown. a The subject reaches for the top of the head (movement 4 ) with the arm extended in front of the body (condition 2). b Same position target as in a with the arm extended along the body (condition 1). $\mathbf{c}$ Same condition as in $\mathbf{b}$, but the subject reaches to the left ear (movement 3 ). Due to the nature of the modulating function $h(\mathbf{x}(t))$, i.e., $h(\mathbf{x}(0))=1$ and $\lim _{t \rightarrow \infty} h(\mathbf{x}(t))=0$ (see the Appendix), the resulting force $\mathbf{F}(\mathbf{x}(t))$ coincides with $\mathbf{v}$ at the beginning of the movement and with $\mathbf{w}$ at the end of the movement,

\section{Discussion}

We have hypothesized that the curvature of unconstrained reaching movements is due to an explicit encapsulation of the task constraints by the CNS in a virtual force field (F2). Movements thus unfold in time according to a dynamical system that attracts the hand to the target position while repelling it from undesirable locations in space (such as objects in the environment, the subject's body and joint limits) and while compensating for unexpected perturbations of the arm. Furthermore, we have argued that the curvature observed in natural movements is not a by-effect of the inherent dynamics of the body but a necessary and voluntarily controlled feature.
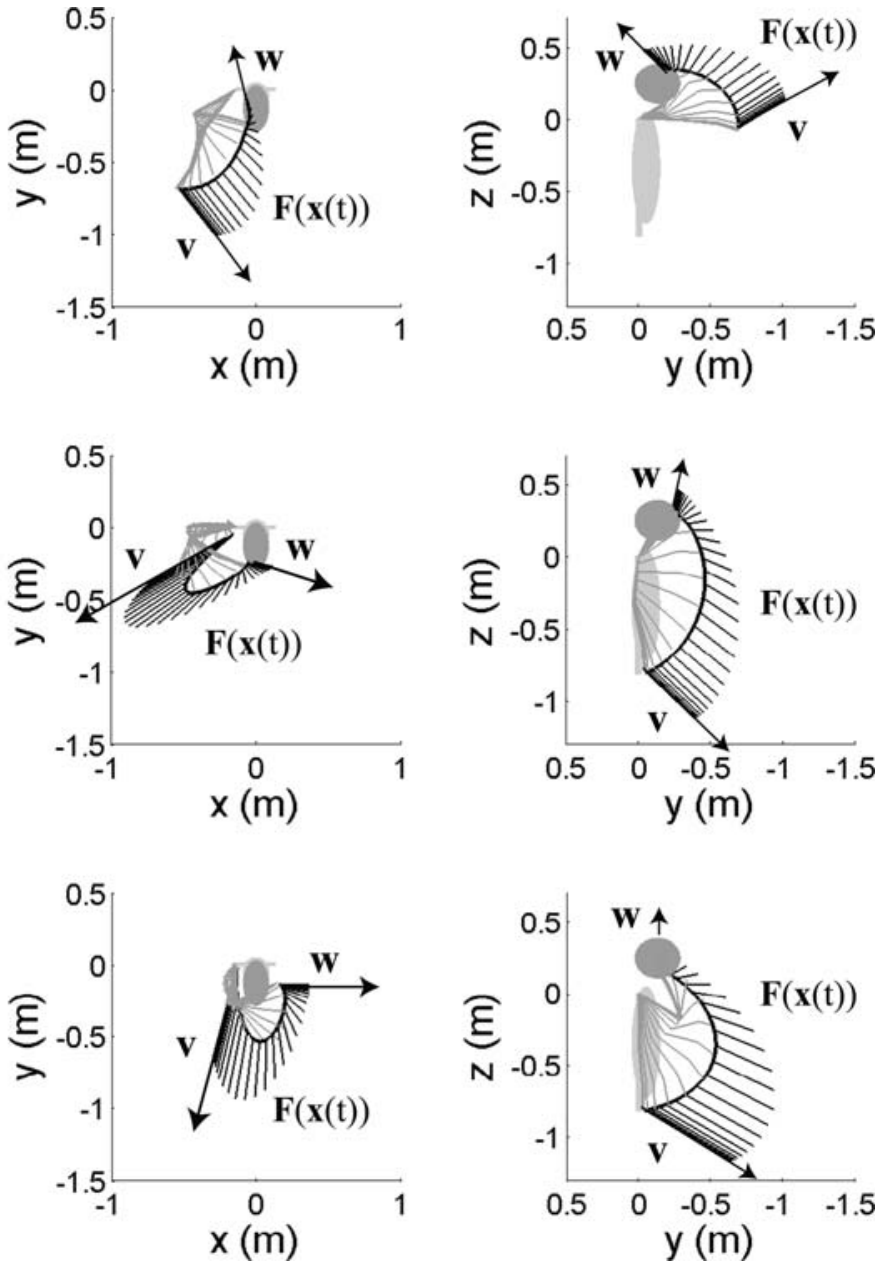

i.e., $\mathbf{F}(t=0)=\mathbf{v}$ and $\mathbf{F}\left(\mathbf{x}=\mathbf{x}^{*}\right)=\mathbf{w}$ (Eq. 3). From $\mathbf{a}$ and $\mathbf{b}$ one can see that the initial condition affects the repulsive forces $\mathbf{v}$ and $\mathbf{w}$. For example, in the second condition (a), $\mathbf{v}$ is in the direction of the target, whereas in the first condition (b and $\mathbf{c}$ ) it is deviated to the right in order to avoid the body and downwards such that the arm does not reach the shoulder extension limit. In addition, $\mathbf{v}$ is coherent within the same condition (see $\mathbf{b}$ and $\mathbf{c}$ ). The target position particularly affects the repulsive force $\mathbf{w}$ (predominant at the end of the movement) that is similar to the normal of the head surface approached. $\mathbf{F}(\mathbf{x}(t))$ was scaled for illustrative reasons

In order to probe our hypothesis, we have conducted motion studies in which healthy adult subjects produced natural reaching motions directed to various locations on their head. To highlight the effect that body constraints may have on the curvature of the movement, we asked the subjects to initiate the movement from two locations: one that required the subject to move alongside the body, the other which allowed the subject to move quasi freely. We showed that our mathematical model, the F2REACH model, could predict the major kinematic features of the movements, such as the bell-shaped velocity profile. Most importantly, it could account for both the weak and strong curvatures of the movements. 


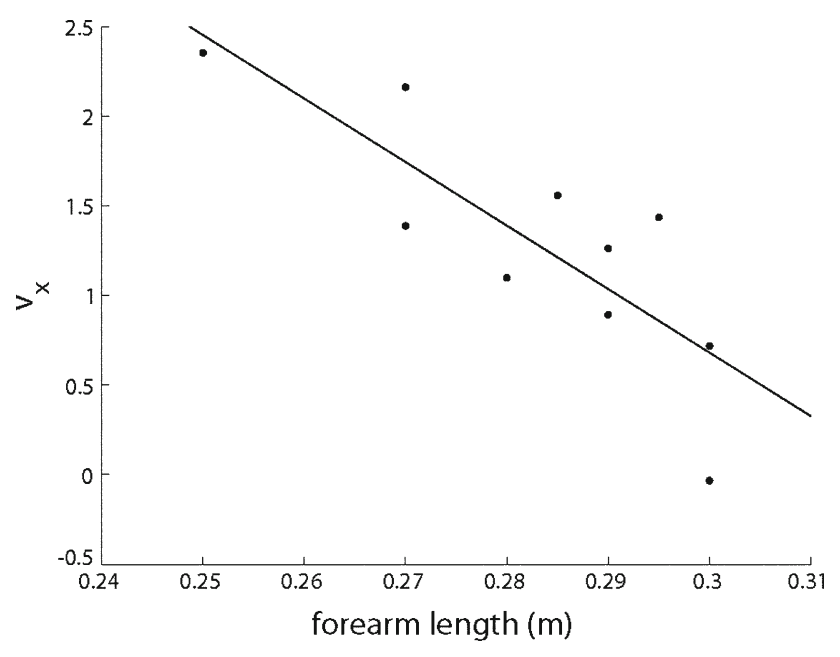

Fig. 12 The amplitude of the repulsive force $\mathbf{w}$ (Eqs. 2 and 3) is linearly correlated to the length of the subjects' forearms for the movement reaching to the left ear with the right arm (condition 1), showing that the repulsive forces in our model are affected by geometrical features of the body. Intuitively, with a shorter forearm, the hand needs to be pushed stronger away to circumvent the head

This led us to argue that a single controller underlies both straight and curved movements. The controller adapts the trajectory according to multiple constraints the subject has consciously or not decided to take into account. Although we have only shown that it can precisely reproduce the kinematics of self-oriented movements, the model is general and can generate natural movements to any target in both intrapersonal and extrapersonal spaces, e.g., in another study that investigates imitation of unnatural postures we successfully use this model to simulate reaching to objects on a table.

\subsection{Assumptions of the model}

The first assumption we have followed is that of a functionally hierarchical motor control system proposed by Bernstein (1947) and translated partly in Bernstein (1996). The hierarchy consists of four levels: complex actions with abstract goals, dealing with 3-dimensional space, muscle synergies, posture and muscle tone. In our study, we considered the first and second levels, in that we addressed 3-dimensional goal-directed reaching movements, characterized by a single target position. By leaving out the question of how such high-level control is then translated into muscle synergies and the control of posture and muscle tone, we follow the observation that: electrical stimulation of the brain motor area elicits reaching movements in primates (Graziano et al. 2002, 2005) and leg movements in frogs (Bizzi et al. 1982). Interestingly, all of these movements converge to the same position in extrinsic space independently from the initial posture. Thus, the control of these movements seems to use solely the definition of the desired final position, and not a description of low-level muscle activations (in a way functionally similar to muscle synergies when compared to activating individual muscles, see d'Avella et al. (2003)). In addition these studies indicate that reaching movements are extensively represented in the motor cortex.

Another argument in favor of a "high-level" extrinsic 3-dimensional representation of movements come from evidence of the many to one mappings between: (1) muscles and joint configurations, (2) muscles and end-effector positions or (3) joint configurations and end-effector positions. Controlling the hand in a 3-dimensional extrinsic space over an intrinsic joint space is advantageous in that it allows to easily encapsulate task constraints, such as avoiding surrounding objects, and plan movements accordingly (these task constraints would have an infinite number of possible representations in the joint and muscle spaces). Also note that we have assumed that movements were computed in a Cartesian frame of reference located on the body. It would however be conceivable to compute the same movement according to a polar coordinate system without affecting the prediction of the model.

The fact that we do not address the above two lowerlevels of motor control, is a limitation of the model. As stated by Bernstein, the problem of translating a kinematic signal encoded in a 3-dimensional extrinsic frame of reference into muscle activations (so-called degrees of freedom problem) is complex because of the redundancy of the muscular system. An infinity of different muscle activations leads to the same kinematic motion of the end-effector. Although this problem is of the highest importance for a complete motor control theory, we do not address this problem here [see d'Avella et al. (2003), Todorov and Jordan (2002) and Guigon et al. (2007) for possible solutions].

Another important assumption we make is that the CNS can represent forces internally. Our model is based upon a force field that encapsulates the constraints of the task, which implies the knowledge of a mapping between different locations in the subject's peripersonal space and virtual repulsive forces. It thus requires the existence of an internal model of the environment in terms of attractive or repulsive force fields in the brain. The above hypotheses are not at odds with the literature. There is substantial evidence that the brain is capable of learning an internal representation of external forces in order to adapt its control of the motion of the hand (Shadmehr and Mussa-Ivaldi 1994; Conditt et al. 1997; Shadmehr and Brashers-Krug 1997; Thoroughman and Shadmehr 2000; Gandolfo et al. 1996), when subjected to these for a long enough period of time. Another force that is centrally represented and integrated in the internal dynamic control models for reaching is the gravitational force (Shadmehr and Mussa-Ivaldi 1994; Papaxanthis et al. 1998). In our model the geometry of the body and external objects, among other factors, contribute to the force field. 
Accordingly, McIntyre et al. (1995) have shown that the brain may integrate an external constraint such as a curved surface through an a priori internal model of the surface geometry.

\subsection{Properties of the model}

Interesting properties of the F2REACH model for motor control are: (i) the system is asymptotically and globally stable; (ii) it exploits a biologically plausible signal-dependant noise and (iii) planning of the movement is done through closed-loop control. This enables on-the-go re-computation of the motion in the face of perturbation or imprecision in the sensory-motor loop. Closed-loop control through afferent and efferent internal feedback loops (Desmurget and Grafton 2000) allows to take into account the uncertainty of the "realworld" instead of just "playing a prerecorded tape" (Todorov 2004). We suggest that only an online mechanism that tightly couples movement planning with movement execution could explain the irregular curvatures observed in some of the trials; the latter were likely due to an on-the-go correction of the trajectory.

Most importantly, we have proposed a force field framework as a powerful mechanism for integrating various constraints related to, e.g., the dynamics and geometry of the arm, external objects and the person's own embodiment, into a unique and generic controller. Whereas the goal of the controller is encoded according to kinematic variables (a position to reach), the constraints are encoded in dynamic variables, the force field, and may as well be expressed in an intrinsic (limit joint angles) or extrinsic (surrounding objects) frame of reference. This framework could reconcile findings that argue for both dynamic and kinematic planning (Vetter et al. 2002; Admiraal et al. 2004), in providing a computational account for how the dynamics of the arm can be taken into account in kinematic planning (Sabes and Jordan 1997). It also explains how external objects might influence the trajectory of the hand (Brenner and Smeets 1995).

Furthermore, the representation of this environmental force field generalizes to performing the motion faster or slower (Harris and Wolpert 1998). This is equivalent to learning to vary the value of the factor $\beta$ in our model (see Eq. 1). Finally the representation of the force field, although local, extends to nearby locations (smoothly decaying away from the position of the perturbation). Similarly, our expression of the force field is spatially continuous.

The extent to which the model's predictions can be generalized to any reaching movement remains to be shown, since we only demonstrated a good agreement of the model with data from reaching movements directed to the head. The movements we have addressed are nevertheless quite generic in that they were entirely unconstrained. For example, we did not observe a reduction of the degrees of freedom as in Klein Breteler et al. (1998) where the subjects had a ten- dency to produce movements in 2D rather than in 3D (see example in Fig. 1). In addition many of the velocity profiles recorded, exhibited asymmetric velocity profiles similar to those observed (Gielen et al. 1985; Brown and Cooke 1990). These characteristics are present in all reaching movements and we are thus confident that the model is generic in its representation of the class of reaching movements. ${ }^{7}$

The force field in our model is parameterized by two constant forces and is thus only an approximation of the real underlying force field. This approximation may lead to imprecisions in the model's predictions, especially in places where the field changes importantly locally.

While our model proposes a way in which the brain may encapsulate all types of motion-related constraints (e.g., body and joint-limits avoidance, inertia of the arm) within a general controller of reaching movements, we do not provide a general method for expressing these constraints in the form of a force field. Our future efforts will concentrate on segmenting the contributions of different constraints and on a mechanism that would allow to learn these through experience.

\subsection{Predictions of the model}

Our model is consistent with several experimental observations and provides a theoretical basis for their interpretation.

For example, in different pointing and reaching studies, systematic misdirections of the fingertip trajectory were observed (de Graaf et al. 1991, 1994; Brenner and Smeets 1995). The misdirections were clockwise and anticlockwise when pointing to targets on the right and on the left frontal space, respectively. To explain their results the authors hypothesized a distorted and contracted internal representation of space (de Graaf et al. 1991, 1994) or speculated that the subjects anticipate the purpose of the target (Brenner and Smeets 1995). Within the repulsive force field framework we propose in this paper, these misdirections are created by the geometrical relationship between the target and the subject's body. ${ }^{8}$ Our model predicts that if one was to repeat the experiment in a different part of the workspace where the misdirections are mainly due to body avoidance, the misdirections would be anticlockwise and clockwise when the target is respectively

\footnotetext{
${ }^{7}$ Current work of ours has applied the model to account for reaching movements oriented to targets on a table in natural and unnatural postures where an artificial constraint is introduced. Preliminary results show that the model again encapsulates with high accuracy all the features of the movements (unpublished data).

8 These two similar studies, de Graaf et al. (1991) and Brenner and Smeets (1995), puzzlingly reported different results. We suggest that the differences observed can be attributed to the distance chosen from the subject to the initial position of the hand $[25 \mathrm{~cm}$ in Brenner and Smeets (1995) and $40 \mathrm{~cm}$ in de Graaf et al. (1991)], as the repulsive forces responsible for avoiding the body would fade away as this distance increases.
} 


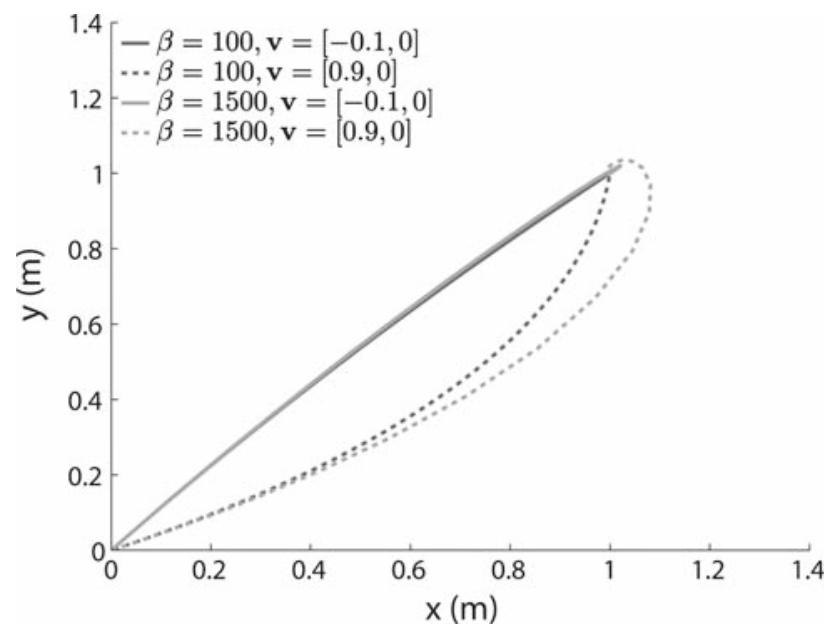

Fig. 13 Prediction of the F2REACH model: the curvature increases with higher speed, here equivalent to higher $\beta$ values. The effect is not visible in quasi-straight movements

right and left from the closest virtual line connecting the subject's trunk with the hand's initial position.

Furthermore, our model predicts that faster movements may be more curved, as shown on Fig. 13. Even though this prediction has been empirically observed (Klein Breteler et al. 1998), it contradicts several experimental and theoretical studies that have shown curvature-speed invariance (Nishikawa et al. 1999; Sha et al. 2006; Liebermann et al. 2008) and suggest that speed and path are planned independently (Todorov and Jordan 2002; Torres and Zipser 2002; Biess et al. 2007). In our model speed modulates the curvature of the path by construction. However, the deviation is also proportional to the magnitude of the repulsive force field such that this effect is particularly important for highly curved movements (see Fig. 13). This might explain why curvature-speed invariance is more consistently observed, as highly curved movements are rarely studied. Otherwise, an additional compensatory mechanism should be added to the model that modulates the force field as a function of desired speed.

Finally, the model suggests that the asymmetry of the velocity profile is due to the difference in directions between the repulsive force field and attracting vector. Finally, even though the curvature of a movement is highly systematic and reproducible (Soechting and Lacquaniti 1981; Pellegrini and Flanders 1996; Admiraal et al. 2004), our model would predict that if you alter the geometry of the subject's body, such as adding a false belly for example, then reaching movements will be displaced away from the artificial object even if this object does not interfere with the original trajectory. Our model also predicts that the shape of the object would matter.

\subsection{Neural correlates of the model}

Most importantly, the F2REACH model we propose is compatible with neurophysiological studies. Primate brain areas have been identified as the loci of the computations involved in the original VITE model (Bullock et al. 1998). Specifically, it was shown that the model's variables display the same dynamics of activation (e.g., response profiles and latency of activity onset) as that of populations of neurons: the hand velocity might be represented in area 4 , whereas the hand acceleration and position in area 5. Note that the extended F2REACH model solicits only quantities that would be easily accessible to the CNS such as distances from the target and initial positions.

A novel feature of the model is the repulsive force field that shapes the landscape of the workspace, meaning that not each position is equally likely to be visited. In other words, the model assumes the existence of neural populations coding for forces related to the body and surrounding objects. Area 5 is a putative region for the computation of the force field, as it receives abundant somatosensory and visual inputs that are necessary for the encapsulation of the geometrical properties of the body and surrounding objects in an internal model (Scott et al. 1997; Graziano et al. 2000). We thus predict the existence of a population of neurons in area 5, whose activity would be close to baseline during straight movements and would rotate in curved movements. In addition, the activation of these neurons would be modulated by the introduction of new objects in the workspace.

\subsection{Conclusion}

We showed that not only the spatial, but also the temporal features of unconstrained and naturally curved reaching movements could be modeled through a dynamical system modulated by a virtual force-field. We found that the model was in very good agreement with kinematic data from human motions, during unconstrained reaching movements directed to the head. We showed that the natural curvature of these movements could be attributed to the interplay between a target attractor and virtual repulsive forces that encapsulate a representation of the geometry of the subject's body. Such a representation is a simple and powerful way to generate kinematically-driven trajectories that comply with the underlying dynamic constraints.

\section{Appendix}

\subsection{Original VITE system}

The original VITE model's dynamics as given by Bullock and Grossberg (1988):

$$
\begin{aligned}
& \dot{\mathbf{y}}(t)=\alpha\left(-\mathbf{y}(t)+\mathbf{x}^{*}(t)-\mathbf{x}(t)\right) \\
& \dot{\mathbf{x}}(t)=\beta t^{\nu} \mathbf{y}(t)
\end{aligned}
$$


where $\mathbf{x}(t)$ corresponds to the current position of the hand in a three-dimensional extrinsic frame of reference and $\mathbf{x}^{*}(t)$ is the location of the target (see Fig. 4). $\mathbf{y}$ is a secondary variable related to the hand velocity. $\alpha$ and $\beta$ are real positive time constants and $v$ is a real positive exponent parameter. The model recomputes at each time step the hand position $\mathbf{x}(t)$, so as to generate an overall straight trajectory to the target that follows a bell-shaped velocity profile. The first term of the equation ensures that the unprimed acceleration vector $\dot{\mathbf{y}}(\mathbf{t})$ is always directed toward the target, i.e., $\mathbf{x}^{*}(\mathbf{t})-\mathbf{x}(t)$, so that the target's position $\mathbf{x}^{*}$ forms a unique attractor of the system. The amplitude of the acceleration $\dot{\mathbf{y}}(\mathbf{t})$ is proportional to the distance separating the hand and the target. $\mathbf{y}(t)$ grows quickly at the beginning of the movement and slows down exponentially towards the end of the movement. To compensate for this asymmetric velocity profile, $\mathbf{y}(t)$ is scaled down in the second equation by a time-dependent variable $\beta t^{\nu} . \dot{\mathbf{x}}(\mathbf{t})$ is the hand's velocity and can be viewed as the output activity of a corresponding neural population that would control agonist muscle motoneurons (Bullock and Grossberg 1988).

\subsection{Nonlinear functions used in the F2REACH model}

The form of the nonlinear function $\mathbf{g}$ in Eq. 1 is the following:

$\mathbf{g}(\mathbf{u})=|\mathbf{d}-\mathbf{u}| \mathbf{u}$

where the control vector $\mathbf{u}(\mathbf{t})=\mathbf{x}^{*}(t)-\mathbf{x}(t)+\boldsymbol{\eta}$ is the vector separating the actual hand position $\mathbf{x}(t)$ from the desired hand position $\mathbf{x}^{*}(t)$ (does not need to be stationary) with signal dependant noise $\eta$. The operator | | stands for the norm of the vector and $\mathbf{d}$ is defined as:

$\mathbf{d}(t)=\mathbf{x}^{*}(t)-\mathbf{x}(0)$

the vector between the target $\mathbf{x}^{*}(t)$ and initial position $\mathbf{x}(0)$, such that the term $|\mathbf{d}-\mathbf{u}|$ is equivalent to the distance separating the actual position of the end-effector from its initial position. $t$ is set to 0 each time a new movement is initiated. In the absence of noise in the control signal $\mathbf{u}$, the multiplicative factor $|\mathbf{d}-\mathbf{u}|$ would be 0 at $t=0$ and no movement would be initiated.

The function $h$ that modulates the force field in Eq. 3 is defined by:

$h(\mathbf{u})=\frac{|\mathbf{u}|}{|\mathbf{u}|+|\mathbf{d}-\mathbf{u}|}$

and normalizes the amplitude of the control signal $\mathbf{u}$.

Acknowledgments This work was funded by the by the Sport and Rehabilitation Engineering Program at EPFL and EU Project IST-2004004370 RobotCub.

\section{References}

Admiraal MA, Kusters MJMAM, Gielen SCAM (2004) Modeling kinematics and dynamics of human arm movements. Motor Control 8(3):312-338

Ajemian R, Bullock D, Grossberg S (2001) A model of movement coordinates in the motor cortex: posture-dependent changes in the gain and direction of single cell tuning curves. Cereb Cortex 11(12):1124-1135

Atkeson CG, Hollerbach JM (1985) Kinematic features of unrestrained vertical arm movements. J Neurosci 5(9):2318-2330

Bernstein NA (1947) On the construction of movements. Medgiz, Moscow

Bernstein NA (1996) Levels of construction of movements. In: Latash ML, Turvey MT (eds) Dexterity and its development. Lawrence Erlbaum Associates, Mahwah, NJ, pp 115-170

Biess A, Liebermann DG, Flash T (2007) A computational model for redundant human three dimensional pointing movements: integration of independant spatial and temporal motor plans simplifies movement dynamics. J Neurosci 27(48):13045-13064

Bizzi E, Accornero N, Chapple W, Hogan N (1982) Arm trajectory formation in monkeys. Exp Brain Res 46(1):139-143

Brenner E, Smeets JBJ (1995) Moving one's finger to a visually specified position: target orientation influences the finger's path. Exp Brain Res 105(2):318-320

Brown SH, Cooke JD (1990) Movement-related phasic muscle activation. I. Relations with temporal profile of movement. J Neurophysiol 63(3):455-464

Bullock D, Grossberg S (1988) Neural dynamics of planned arm movements: emergent invariants and speed-accuracy properties during trajectory formation. Psychol Rev 95(1):49-90

Bullock D, Grossberg S, Mannes C (1993) A neural network model for cursive script production. Biol Cybern 70(1):15-28

Bullock D, Cisek P, Grossberg S (1998) Cortical networks for control of voluntary arm movements under variable force conditions. Cereb Cortex 8(1):48-62

Christel MI, Billard A (2002) Comparison between macaques' and humans' kinematics of prehension: the role of morphological differences and control mechanisms. Behav Brain Res 131(1-2):169184

Cisek P, Grossberg S, Bullock D (1998) A cortico-spinal model of reaching and proprioception under multiple task constraints. J Cogn Neurosci 10(4):425-444

Clamman HP (1969) Statistical analysis of motor unit firing pattern in human skeletal muscle. Biophys J 9:1233-1251

Clancy EA, Hogan N (1999) Probability density of the surface electromyogram and its relation to amplitude detectors. IEEE Trans Biomed Eng 46(6):730-739

Conditt MA, Gandolfo F, Mussa-Ivaldi FA (1997) The motor system does not learn the dynamics of the arm by rote memorization of past experience. J Neurophysiol 78(1):554-560

Cruse H, Brüwer M (1987) The human arm as a redundant manipulator: the control of path and joint angles. Biol Cybern 57(1-2):134-144

d'Avella A, Saltiel P, Bizzi E (2003) Combinations of muscle synergies in the construction of a natural motor behavior. Nat Neurosci 6(3):300-308

de Graaf JB, Sittig AC, Denier von der Gon JJ (1991) Misdirections in slow goal-directed arm movements and pointer-setting tasks. Exp Brain Res 84(2):434-438

de Graaf JB, Sittig AC, Denier von der Gon JJ (1994) Misdirections in slow, goal-directed arm movements are not primarily visually based. Exp Brain Res 99(3):464-472

Dalby JT, Gibson D, Grossi V, Schneider RD (1980) Lateralized hand gesture during speech. J Motor Behav 12(4):292-297

De Renzi E, Lucchelli F (1988) Ideational apraxia. Brain 111(Pt 5): $1173-1185$ 
Desmurget M, Grafton S (2000) Forward modeling allows feedback control for fast reaching movements. Trends Cogn Sci 4(11):423431

Desmurget M, Jordan M, Prablanc C, Jeannerod M (1997) Constrained and unconstrained movements involve different control strategies. J Neurophysiol 77(3):1644-1650

Fitts PM (1954) The information capacity of the human motor system in controlling the amplitude of movement. J Exp Psychol 47(6):381391

Flash T (1987) The control of hand equilibrium trajectories in multijoint arm movements. Biol Cybern 57(4-5):257-274

Flash T, Hogan N(1985) The coordination of arm movements: an experimentally confirmed mathematical model. J Neurosci 5(7):1688-1703

Gandolfo F, Mussa-Ivaldi FA, Bizzi E (1996) Motor learning by field approximation. Proc Natl Acad Sci USA 93(9):3843-3846

Gielen CCAM, van den Oosten K, Pull ter Gunne F (1985) Relation between EMG activation patterns and kinematic properties of aimed arm movements. J Mot Behav 17(4):421-442

Goldenberg G, Hagmann S (1997) The meaning of meaningless gestures: a study of visuo-imitative apraxia. Neuropsychologia 35(3):333-341

Graziano MSA, Cooke DF, Taylor CSR (2000) Coding the location of the arm by sight. Science 290(5497):1782-1786

Graziano MSA, Taylor CSR, Moore T (2002) Complex movements evoked by microstimulation of precentral cortex. Neuron 34(5):841-851

Graziano MSA, Aflalo TNS, Cooke DF (2005) Arm movements evoked by electrical stimulation in the motor cortex of monkeys. J Neurophysiol 94(6):4209-4223

Guigon E, Baraduc P, Desmurget M (2007) Computational motor control: redundancy and invariance. J Neurophysiol 97(1):331-347

Hamilton AFC, Wolpert DM (2002) Controlling the statistics of action: obstacle avoidance. J Neurophysiol 87(5):2434-2440

Harris CM, Wolpert DM (1998) Signal-dependent noise determines motor planning. Nature 394(6695):780-784

Hersch M, Billard A (2007) Reaching with multi-referential dynamical systems. Auton Robot 25(1-2):71-83

Hoos H, Stützle T (2004) Stochastic local search: Foundations and applications. Elsevier/Morgan Kaufmann, San Francisco

Klein Breteler MD, Meulenbroek RGJ, Gielen SCAM (1998) Geometric features of workspace and joint-space paths of 3D reaching movements. Acta Psychol 100(1-2):37-53

Lacquaniti F, Terzuolo C, Viviani P (1983) The law relating the kinematic and figural aspects of drawing movements. Acta Psychol 54(1-3):115-130

Lacquaniti F, Soechting JF, Terzuolo SA (1986) Path constraints on point-to-point arm movements in three-dimensional space. Neuroscience 17(2):313-324

Lavergne J, Kimura D (1987) Hand movement asymmetry during speech: no effect of speaking topic. Neuropsychologia 25(4): 689-693

Liebermann DG, Biess A, Gielen CCAM, Flash T (2006) Intrinsic joint kinematic planning. II: Hand-path predictions based on a Listing's plane constraint. Exp Brain Res 171(2):155-173

Liebermann DG, Krasovsky T, Berman S (2008) Planning maximally smooth hand movements constrained to nonplanar workspaces. J Mot Behav 40(6):516-531

Matthews PBC (1996) Relationship of firing intervals of human motor units to the trajectory of post-spike after-hyperpolarization and synaptic noise. J Physiol 492(Pt 2):597-628

McIntyre J, Gurfinkel EV, Lipshits MI, Droulez J, Gurfinkel VS (1995) Measurements of human force control during a constrained arm motion using a force-actuated joystick. J Neurophysiol 73(3):1201-1222
Miall RC, Haggard PN (1995) The curvature of human arm movements in the absence of visual experience. Exp Brain Res 103(3):421-428

Moran DW, Schwartz AB (1999) Motor cortical activity during drawing movements: population representation during spiral tracing. J Neurophysiol 82(5):2693-2704

Morasso P (1981) Spatial control of arm movements. Exp Brain Res 42(2):223-227

Nakano E, Imamizu H, Osu R, Uno Y, Gomi H, Yoshioka T, Kawato M (1999) Quantitative examinations of internal representations for arm trajectory planning: minimum commanded torque change model. J Neurophysiol 81(5):2140-2155

Neter J, Kutner MH, Nachtsheim CJ, Wasserman W (1996) Applied linear statistical models. McGraw-Hill/Irwin, Chicago

Nishikawa KC, Murray ST, Flanders M (1999) Do arm postures vary with the speed of reaching? J Neurophysiol 81(5):2582-2586

Okadome T, Honda M (1999) Kinematic construction of the trajectory of sequential arm movements. Biol Cybern 80(3):157-169

Oldfield RC (1971) The assessment and analysis of handedness: the Edinburgh inventory. Neuropsychologia 9(1):97-113

Osu R, Uno Y, Koike Y, Kawato M (1997) Possible explanations for trajectory curvature in multijoint arm movements. J Exp Psychol Hum Percept Perform 23(3):890-913

Osu R, Kamimura N, Iwasaki H, Nakano E, Harris CM, Wada Y, Kawato M (2004) Optimal impedance control for task achievement in the presence of signal-dependant noise. J Neurophysiol 92(2):1199-1215

Paine RW, Grossberg S, Van Gemmert AWA (2004) A quantitative evaluation of the AVITEWRITE model of handwriting learning. Hum Mov Sci 23(6):837-860

Papaxanthis C, Pozzo T, Popov KE, McIntyre J (1998) Hand trajectories of vertical arm movements in one-G and zero-G environments. Evidence for a central representation of gravitational force. Exp Brain Res 120(4):496-502

Pellegrini JJ, Flanders M (1996) Force path curvature and conserved features of muscle activation. Exp Brain Res 110(1):80-90

Petreska B, Adriani M, Blanke O, Billard AG (2007) Apraxia: a review. Prog Brain Res 164:61-83

Sabes PN, Jordan MI (1997) Obstacle avoidance and a perturbation sensitivity model of motor planning. J Neurosci 17(18):7119-7128

Sauser E, Billard A (2006) Dynamic updating of distributed neural representations using forward models. Biol Cybern 95(6):567-588

Schaal S, Sternad D (2001) Origins and violations of the $2 / 3$ power law in rhythmic three-dimensional arm movements. Exp Brain Res 136(1):60-72

Schmidt RA, Zelaznik H, Hawkins B, Frank JS, Quinn JT (1979) Motor-output variability: a theory for the accuracy of rapid motor acts. Psychol Rev 47(5):415-451

Scott SH, Sergio LE, Kalaska JF (1997) Reaching movements with similar hand paths but different arm orientations. II. Activity of individual cells in dorsal premotor cortex and parietal area 5 . J Neurophysiol 78(5):2413-2426

Sha D, Patton J, Mussa-Ivaldi FA (2006) Minimum jerk reaching movements of human arm with mechanical constraints at endpoint. Int J Comput Syst Signal 7(1):41-50

Shadmehr R, Brashers-Krug T (1997) Functional stages in the formation of human long-term motor memory. J Neurosci 17(1): 409-419

Shadmehr R, Mussa-Ivaldi FA (1994) Adaptive representation of dynamics during learning of a motor task. J Neurosci 14(5 Pt 2): 3208-3224

Soechting JF, Lacquaniti F (1981) Invariant characteristics of a pointing movement in man. J Neurosci 1(7):710-720

Soechting JF, Buneo CA, Herrmann U, Flandres M (1995) Moving effortlessly in three dimensions: does Donders' law apply to arm movement? J Neurosci 15(9):6271-6280 
St-Amant Y, Rancourt D, Clancy EA (1998) Influence of smoothing window length on electromyogram amplitude estimates. IEEE Trans Biomed Eng 45(6):795-800

Sutton GG, Sykes K (1967) The variation of hand tremor with force in healthy subjects. J Physiol 191(3):699-711

Thoroughman KA, Shadmehr R (2000) Learning of action through adaptive combination of motor primitives. Nature 407(6805):742747

Todorov E (2004) Optimality principles in sensorimotor control. Nat Neurosci 7(9):907-915

Todorov E, Jordan MI (2002) Optimal feedback control as a theory of motor coordination. Nat Neurosci 5(11):1226-1235

Torres EB, Zipser D (2002) Reaching to grasp with a multi-joined arm. I. Computational model. J Neurophysiol 88(5):2355-2367

Uno Y, Kawato M, Suzuki R (1989) Formation and control of optimal trajectory in human multijoint arm movement. Minimum torquechange model. Biol Cybern 61(2):89-101
Vetter P, Flash T, Wolpert DM (2002) Planning movements in a simple redundant task. Curr Biol 12(6):488-491

Wada Y, Kaneko Y, Nakano E, Osu R, Kawato M (2001) Quantitative examinations for multi joint arm trajectory planning-using a robust calculation algorithm of the minimum commanded torque change trajectory. Neural Netw 14(4-5):381-393

Wang W, Chan SS, Heldman DA, Moran DW (2007) Motor cortical representation of position and velocity during reaching. J Neurophysiol 97(6):4258-4270

Wolpert DM, Ghahramani Z, Jordan MI (1994) Perceptual distortion contributes to the curvature of human reaching movements. Exp Brain Res 98(1):153-156

Wolpert DM, Ghahramani Z, Jordan MI (1995) Are arm trajectories planned in kinematic or dynamic coordinates? An adaptation study. Exp Brain Res 103(3):460-470 